\title{
1 Hyperactive end joining repair mediates resistance to DNA damaging therapy in p53-
} 2 deficient cells

Rashmi J. Kumar ${ }^{1,2}$, Hui Xiao Chao ${ }^{3}$, Victoria R. Roberts ${ }^{1}$, Aurora R. Sullivan ${ }^{1}$, Sonam J. Shah ${ }^{1}$,

6 Dennis A. Simpson ${ }^{1}$, Wanjuan Feng ${ }^{1}$, Anne-Sophie Wozny ${ }^{1}$, Sunil Kumar ${ }^{1}$, Jeremy E. Purvis ${ }^{3,4}$,

7 Gaorav P. Gupta ${ }^{1,2,5,6, \#}$

$8 \quad{ }^{1}$ Lineberger Comprehensive Cancer Center, University of North Carolina at Chapel Hill, Chapel

9 Hill, NC, 27599, USA

$10{ }^{2}$ Curriculum in Genetics and Molecular Biology, University of North Carolina at Chapel Hill,

11 Chapel Hill, NC, 27599, USA

$12{ }^{3}$ Curriculum in Bioinformatics and Computational Biology, University of North Carolina at Chapel

13 Hill, Chapel Hill, NC, 27599, USA

$14{ }^{3}$ Department of Computational Medicine, University of North Carolina at Chapel Hill, Chapel Hill, 15 NC, 27599, USA

$16{ }^{4}$ Department of Genetics, University of North Carolina at Chapel Hill, Chapel Hill, NC, 27599,

17 USA

$18{ }^{5}$ Department of Radiation Oncology, University of North Carolina at Chapel Hill, Chapel Hill, NC, 19 27599, USA

$20{ }^{6}$ Department of Biochemistry and Biophysics, University of North Carolina at Chapel Hill, Chapel 21 Hill, NC, 27599, USA

\#Correspondence:

Gaorav P. Gupta, MD PhD

27 Assistant Professor

28 Department of Radiation Oncology

29 Department of Biochemistry and Biophysics

30 University of North Carolina at Chapel Hill

31 Chapel Hill, NC 27599

32 gaorav_gupta@med.unc.edu 


\section{Abstract}

34 TP53 mutations in cancer are associated with poor patient outcomes and resistance to

35 DNA damaging therapies ${ }^{1-3}$. However, the mechanisms underlying treatment resistance

36 in p53-deficient cells remain poorly characterized. Here, we show that p53-deficient cells

37 exhibit hyperactive repair of therapy-induced DNA double strand breaks (DSBs), which is

38 suppressed by inhibition of DNA-dependent protein kinase (DNA-PK). Single-cell

39 analyses of DSB repair kinetics and cell cycle state transitions reveal an essential role for

40 DNA-PK in suppressing $\mathrm{S}$ phase DNA damage and mitotic catastrophe in p53-deficient

41 cells. Yet, a subset of p53-deficient cells exhibit intrinsic resistance to therapeutic DSBs

42 due to a repair pathway that is not sensitive to DNA-PK inhibition. We show that p53

43 deficiency induces overexpression of DNA Polymerase Theta (Pol $\theta$ ), which mediates an

44 alternative end-joining repair pathway that becomes hyperactivated by DNA-PK

45 inhibition ${ }^{4}$. Combined inhibition of DNA-PK and Pol $\theta$ restores therapeutic DNA damage

46 sensitivity in p53-deficient cells. Thus, our study identifies two targetable DSB end joining

47 pathways that can be suppressed as a strategy to overcome resistance to DNA-damaging

48 therapies in p53-deficient cancers.

\section{Introduction}

TP53 is the most commonly mutated tumor suppressor gene ${ }^{5}$. p53 mediates pleiotropic tumor suppressive effects through regulation of cell cycle arrest, apoptosis, and cellular metabolism in response to cellular stress ${ }^{6,7}$. Beyond its role as a tumor suppressor, loss of functional p53 is associated with poor prognostic outcomes across many different cancer types ${ }^{1,8-10}$. There is both clinical and preclinical evidence that p53deficient cancers exhibit resistance to a variety of DNA damaging therapies ${ }^{2,3,11-14}$. 
The mechanisms for therapeutic resistance in p53-deficient cells remains poorly characterized. Past work has suggested a role for loss of p53-mediated apoptosis ${ }^{12,15}$.

59 However, the response of epithelial cancer cells to DNA damaging therapy is often

60 determined by the efficiency of inducing senescence or mitotic catastrophe, rather than

61 apoptosis ${ }^{16,17}$. p53 is also a transcription factor that responds to DNA double strand

62 breaks (DSBs) to determine cellular fate ${ }^{7,18}$. Recent insights have revealed the

63 importance of p53-signaling waves in regulation of cellular fate decisions of quiescence

64 versus cell cycle re-entry after DNA damage ${ }^{19,20}$. However, the mechanisms that

65 determine such cell fate decisions upon DNA damage induction in p53-mutant epithelial

66 cells have not been established, and may lead to novel strategies to restore treatment

67 sensitivity.

In this study, we investigate altered DNA repair mechanisms in p53 deficiency as

69 a major contributor to resistance to DNA damaging therapies. We find that p53-deficient

70 cells exhibit hyperactive repair and accelerated resolution of DNA damage foci. Utilizing

71 live-cell imaging, we show that this ability to resolve DNA damage rapidly is partially

72 dependent on DNA-PK, a critical serine/threonine kinase in the non-homologous end

73 joining (NHEJ) pathway ${ }^{21}$. Inhibition of DNA-PK using the small molecule inhibitor

74 NU7441 partially sensitizes p53-deficient cells to DSB inducing agents. We further show

75 that this effect is specifically due to propagation of $S$ phase related damage leading to

76 mitotic catastrophe, highlighting a role for DNA-PK in S phase DNA damage repair that

77 was previously under appreciated. Furthermore, using chromosomal break repair assays

78 we show that in the context of inhibitor treatment, some p53-deficient cells utilize

79 alternative end-joining repair in a compensatory manner to escape cell death. Thus, our 
80 work provides critical insight into a clinically-relevant mechanism for why p53-deficient

81 cells are resistant to DNA damaging therapies.

82 Results

83 p53-deficient cells exhibit radioresistance and accelerated resolution of DNA DSBs

We first established an isogenic cell system to investigate determinants of

85 treatment-induced cell fate in p53-deficient cells. In order to minimize potential contributions of accessory mutations on phenotypes observed in cancer cell line models,

87 we used CRISPR/Cas9 to disrupt TP53 in the p53-proficient immortalized epithelial cell

88 line model hTert-RPE1 ("RPE1"), which has also been a preferred model for investigating

89 p53-dependent cell fate ${ }^{18,20,22}$. Two independent CRISPR/Cas9-targeted TP53- RPE1

90 clones were selected for further study after confirming cells were deficient for p53 protein

91 and lacked p53-dependent transcriptional induction of p21 in response to ionizing

92 radiation (IR) (Supplementary Fig. 1a-c).

93 To assess whether p53 deficiency confers a proliferative advantage when treated

94 with ionizing radiation, we performed a mixed competition assay. We took mCherry

95 labelled RPE1 and mixed them with equal numbers of unlabeled TP53-- RPE1 or p53-

96 proficient RPE1 (control) (Fig. 1a). We quantified the relative abundance of the unlabeled

97 cells after to exposure to IR (0 - 6Gy), normalized to untreated samples at each timepoint.

98 RPE1 labeled and unlabeled cells maintained stable representation across time and

99 treatment conditions (Supplementary Fig. 1d). Additionally, p53-deficient cells did not

100 demonstrate a proliferation advantage in the absence of RT. However, treatment with IR

101 at any dose level led to substantial positive selection for p53-deficient cells(Fig. 1b,c).

102 We also observed that p53 deficiency induced resistance to the radiomimetic clastogen, 
103 Neocarzinostatin (NCS) by colony forming assay (Supplementary Fig. 1e-i). Thus p53

104 deficiency in this isogenic model is sufficient to induce radioresistance.

105 Unrepaired DSBs can suppress proliferation through the engagement of DNA

106 damage-induced cell cycle checkpoints. We examined kinetics of DSB repair by

107 performing immunofluorescence for 53BP1 and $\gamma \mathrm{H} 2 \mathrm{AX}$ after treatment of p53 WT and

108 TP53 $^{-/}$cells with 5Gy IR (Fig. 1d,e and Supplementary Fig. 1j,k). We observed a

109 reduction in the number of 53BP1 damage foci in TP53-- cells as early as 30 minutes

110 after treatment, that became even more pronounced by 4 hours post-treatment (Fig.

$1111 \mathrm{~d}, \mathrm{e})$. Similar patterns of reduced foci formation were also apparent with $\gamma \mathrm{H} 2 \mathrm{AX}$ staining

112 at early timepoints (Supplementary Fig. 1j,k). Quantification of IR-induced DSBs by

113 neutral COMET assay revealed an equivalent DSB burden induced immediately after 5Gy

$114 \mathrm{IR}$, irrespective of p53 status (Fig. 1f,g). However, by 4 hours post-treatment, tail DNA

115 percent was significantly reduced in the TP53-- cells while remaining elevated in p53-

116 proficient RPE1 (Fig. 1f,g). Thus, p53 deficiency is sufficient to induce radioresistance

117 and accelerated DSB repair in an isogenic model.

118 Inhibition of DNA-PK restores DNA damage foci formation in p53-deficient cells

119 To directly assess the relationship between DSB repair kinetics, cell cycle status,

120 and cell fate at the single cell level, we established a live cell imaging platform (Fig. 2a).

121 RPE1 cells were dually labeled with PCNA-mCherry (to monitor cell cycle state

122 transitions) and 53BP1-mVenus (to monitor DSB foci kinetics) (Fig. 2b) ${ }^{23,24}$. These dual

123 labeled cells were treated with scrambled siRNA (si-Control) or siRNA targeting TP53

124 (si-TP53), the latter of which resulted in $>90 \%$ knockdown of TP53 transcript and

125 elimination of p53-dependent CDKN1A transcription in response to IR (Fig. 2c). 48 hours 
126 after siRNA treatment, RPE1 cells were imaged for a total of 72 hours every 10 minutes,

127 and 18 hours into imaging, the DSB inducing agent was added (Fig. 2a). To minimize

128 time from radiation exposure to image capture and to induce equivalent DSBs in each

129 population of cells, we utilized $100 \mathrm{ng} / \mathrm{ml}$ of Neocarzinostatin (NCS), a well-known radio-

130 mimetic. NCS has been previously utilized in studies evaluating DNA DSB repair in

131 conjunction with live-cell imaging and has been shown to induce peak DSBs within 10

132 minutes of drug addition ${ }^{25,26}$. This experimental design allowed us to determine the cell

133 cycle status of each cell within the asynchronous cell population at the time of NCS

134 exposure. After NCS treatment, single-cell analyses for DSB repair foci kinetics and cell

135 cycle outcomes were performed. As anticipated from Fig. 1b,c, analysis of global

136 proliferation by live-cell imaging revealed significantly greater proliferation of p53-deficient

137 RPE1 cells relative to controls after NCS treatment (Supplementary Fig. 2a,b).

To analyze DSB repair kinetics in cells exposed to NCS, we tracked and quantified

139 53BP1 foci in single cells and plotted heatmaps of damage foci burden over time from

140 cell birth to mitosis (Fig. 2d). Our results indicate that cells with functional p53 sustain

141 high levels of damage foci in a prolonged manner after NCS exposure. In contrast, p53-

142 deficient cells developed a lower peak burden of 53BP1 foci after NCS treatment, with

143 accelerated resolution of damage foci to baseline levels (Fig. 2d,f). Given the rapidity with

144 which 53BP1 foci were being resolved, we hypothesized that hyperactive NHEJ may be

145 contributing. We thus performed the same experiment in the presence of an inhibitor of

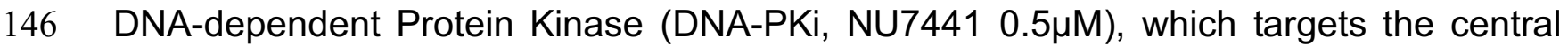

147 kinase in the NHEJ pathway ${ }^{27-29}$. Strikingly, DNA-PKi qualitatively abolished the

148 difference in 53BP1 kinetics after NCS treatment between p53-deficient and proficient 
149 cells (Fig. 2e). To quantitatively assess the magnitude in damage burden, we calculated

150 peak maximum 53BP1 foci values for each cell represented in the heatmap (Fig. 2f).

151 Consistent with the heatmap representation, the median peak foci count after NCS

152 treatment was $40 \%$ lower in si-TP53 treated cells relative to controls (Fig. 2f, p<0.0001).

153 Notably, DNA-PKi treatment resulted in a >2-fold increase in peak 53BP1 foci levels in

154 the p53-deficient cells, whereas there was no comparable effect in control cells (Fig. 2f).

155 These results indicate that DNA-PK activity is required for accelerated resolution of

156 clastogen-induced DNA damage foci in p53-deficient cells.

Following this analysis, we studied the effects of DNA-PKi in different phases of

158 the cell cycle during drug exposure. We used PCNA live-cell imaging to resolve cell cycle

159 phase transitions in cells tracked for 53BP1 foci kinetics. We performed area under the

160 curve (AUC) analyses in single cells to estimate total DNA damage burden during G1 and

161 S phase after NCS exposure (Fig. 2g). This analysis revealed that the diminished 53BP1

162 foci burden observed in p53-deficient cells was most pronounced during $\mathrm{S}$ phase relative

163 to control cells (Fig. 2g). DNA-PKi treatment significantly increased S phase 53BP1

164 burden in both si-Control and si-TP53 treated RPE1 cells (Fig. 2g). While si-TP53 treated

165 cells in G1 were also affected to a lesser degree, we were curious to examine if the effect

166 was in part due to loss of the p53-dependent G1/S checkpoint resulting in propagation of

167 unrepaired DNA damage into S phase. Indeed, we found that DNA-PKi induced a drastic

168 increase in 53BP1 foci as p53-deficient cells transitioned from G1 to S phase, which

169 subsequently diminished over time (Fig. $2 \mathrm{~h}, \mathrm{p}<0.00001$ at $\mathrm{t}=$ start of $\mathrm{S}$ phase). Thus,

170 DNA-PK is required for hyperactive resolution of clastogen-induced DSB foci in p53-

171 deficient cells, and most prominently during S phase. 
Checkpoint responses halt p53-proficient cells upon exposure to NCS while p53-

To investigate the association between DNA damage and activation of cell cycle

175 checkpoints, we quantified cell cycle phase durations for all treatment conditions (Fig.

$1763 a, b)$. p53-proficient G1 cells exposed to NCS induced a significant prolongation of G1,

177 indicative of G1/S checkpoint activation, with a substantial proportion of cells remaining 178 arrested for the duration of imaging (Fig. 3c and Supplementary Fig. 3a-c). Similarly, cells

179 exposed to NCS in S phase exhibited a G2-M checkpoint (Fig. 3d). p53-deficient cells

180 exhibited no prolongation of G1 duration after NCS, consistent with the notion that G1/S

181 checkpoint activation is p53-dependent (Fig. 3e) $)^{30,31}$. DNA-PK inhibition did not alter G1

182 duration in either p53-proficient or p53-deficient cells (Fig. 3c,e). In contrast, DNA-PKi

183 increased the duration of G2-M checkpoints irrespective of p53 status (Fig. 3d,f). These

184 observations suggest that increased levels of S phase DNA damage induced by DNA-

185 PKi and NCS treatment (see Fig. 2g,h) result in activation of a G2/M checkpoint that is,

186 at least partially, p53-independent. However, the duration of G2/M checkpoint activation

187 differed by p53 status. While p53-proficient cells frequently remained arrested for the

188 entire duration of imaging (open circles, Fig. 3c,d), p53-deficient cells experienced a more

189 transient prolongation of G2 duration followed by progression into mitosis (Fig. 3e,f).

190 Inhibition of DNA-PK induces catastrophic mitoses in p53-deficient cells

191 We next used a heatmap representation to track the fate of individual cells from

192 birth until mitosis (Fig. 4a,b, top panels). Red bars indicate a mitotic catastrophe or

193 apoptosis event (Supplementary Fig. 4a,b). The median cell cycle time for both untreated

194 p53-proficient and p53-deficient cells was approximately 22-24 hours. NCS treatment is 
195 indicated as a dashed line at the 18 hour timepoint. Individual cells are ordered according

196 to cell cycle phase at the time of NCS treatment (G1 versus S) and eventual cell fate

197 (viable, G1 arrest, G2 arrest, or mitotic catastrophe/apoptosis). The majority (70\%) of p53-

198 proficient (si-Control) G1 cells exposed to NCS activated a G1 checkpoint that was

199 maintained for the remainder of imaging (Fig. 4a). 26\% of these cells underwent G2 arrest

200 or mitotic catastrophe, whereas only $3 \%$ retained their proliferative capacity (Fig. $4 a$ ).

201 Control cells exposed to NCS in S phase exhibited more diverse cell fates: 40\% G2 arrest,

$20217 \%$ mitotic catastrophe, and $43 \%$ that retained proliferative capacity. These

203 observations, made using single-cell tracking of asynchronous cell populations, are

204 consistent with observations of intrinsic radioresistance of S phase cells using cell

205 synchronization methods ${ }^{32}$. In contrast, the majority of p53-deficient (i.e., si-TP53

206 treated) cells in G1 or S at the time of NCS treatment remained viable without perceptible

207 engagement of any cell cycle checkpoints (Fig. 4b, 80\% and 87\%, respectively).

208 Consistent with prior 53BP1 analyses, S phase cells are most sensitized to DNA-PKi as

209 the addition of the inhibitor increased G2 arrest frequency in control cells (40\% to $91 \%$ ),

210 and increased mitotic catastrophe in p53-deficient cells (13\% to 47\%, Fig. 4a,b). In total,

211 the percentage of viable p53-deficient cells after NCS decreased from $87 \%$ to $47 \%$ when

212 treated in S phase with DNA-PK inhibition ( $p<0.0001$, Fisher's exact test).

213 Despite the significant increase in mitotic catastrophe induced by combined

214 treatment with DNA-PKi and NCS, 47\% of p53-deficient cells exhibit intrinsic resistance

215 to therapy with retained proliferative viability (Fig. 4b). We hypothesized that levels of

216 unrepaired DNA damage may be determinants of viable (i.e., resistant) versus non-viable

217 (i.e., sensitive) cell fates. To evaluate this hypothesis, we quantified integral DNA damage 
218 burden in p53-deficient RPE1 with viable versus non-viable mitotic outcomes (Fig. 4c and

219 Supplementary Fig. 4c). The mean integral DNA damage burden was approximately 2-

220 fold higher in non-viable cells, relative to cells that viably completed mitosis $(p<0.0001)$.

221 Further analysis revealed that integral DNA damage burden in S phase was most highly

222 associated with cell viability after drug treatment (Supplementary Fig. 4c). In addition, we

223 traced the average 53BP1 foci burden over time for these two cohorts (Fig. 4d). Our

224 results indicate that cells with non-viable mitotic outcomes have an increased peak value

225 of DNA damage after treatment with DNA-PKi and NCS, which remains elevated over

226 time $(p<0.0001$ at $t=20$ hrs, Fig. 4d). Conversely, these findings indicate that p53-

227 deficient cells that exhibit intrinsic therapeutic resistance may be utilizing compensatory

228 DSB repair pathways to counteract the effects of NCS and DNA-PKi prior to mitotic entry.

229 p53-deficient cells utilize alternative end-joining pathways in the absence of active

230 DNA-PK

231 Prior studies have demonstrated that cells with NHEJ deficiency exhibit a

232 compensatory increase in alternative end-joining repair mediated by DNA polymerase

233 theta (Pol $\theta$, gene $P O L Q)^{4,33,34}$. Polymerase theta dependent end joining (TMEJ) of DNA

234 DSBs is characterized by deletions and templated insertions that are flanked by short

235 tracts of sequence identity, or microhomology $(\mathrm{MH})^{4}$. We found that $P O L Q$ expression

236 was 10- to 20-fold higher in two independent TP53-- RPE1 clones, relative to parental

237 TP53 wild-type cells (Fig. 5a). POLQ is also overexpressed in TCGA breast, lung,

238 bladder, colorectal, gastric, glioblastoma, pancreatic, prostate, melanoma, and uterine

239 cancers with TP53 mutation, relative to their TP53 wild-type counterparts (Fig. 5b). 
To assess whether hyperactive TMEJ contributes to therapeutic resistance of

241 TP53 $^{--}$RPE1 cells to NCS and DNA-PKi, we sought to inhibit Pol $\theta$. As pharmacological

242 inhibitors of Pol $\theta$ are not yet commercially available, we created a double knockout

243 POLQ ${ }^{-/}$TP53\%- RPE1 line (Supplementary Fig. 5a). Bi-allelic frameshift mutations in

$244 P O L Q$ were confirmed by Sanger sequencing and functional deficiency was established

245 using an extrachromosomal TMEJ repair assay (Supplementary Fig. 5b-d) ${ }^{4}$.

246 To directly assess whether TMEJ repair is increased after DNA-PKi treatment, we

247 analyzed chromosomal break repair patterns at a site-specific DSB in p53-deficient RPE1

248 cells. Cells were transfected with Cas9 ribonucleoprotein (RNP) complexes that target 249 the LBR locus, with or without DNA-PKi ${ }^{35}$. Genomic DNA was harvested 60 hours later

250 and analyzed for break repair patterns using next generation sequencing (NGS) (Fig. 5c).

251 Target amplification and TIDE analyses confirmed high rates of target site cleavage in all

252 samples transfected with a full complement of Cas9-RNP (Supplementary Fig. 5e,f) ${ }^{36}$.

253 We applied a bioinformatic algorithm (ScarMapper, see methods) to characterize the 254 spectrum of repair products with at least $0.1 \%$ prevalence, classified according to the size 255 of left deletion (LD), right deletion (RD), insertion (Ins), and microhomology (MH) 256 (ScarMapper Methods). Indels <5bp were categorized as NHEJ, with the predominant 257 repair product being a $+\mathrm{A} 1 \mathrm{bp}$ insertion ${ }^{35}$. TMEJ was defined as repair products whose 258 frequency was diminished by at least 2-fold in $P O L Q^{-/-}$cells. All other repair products 259 were categorized as "Unclassified." DNA-PK inhibition in TP53\%- RPE1 cells results in a 260 substantial reduction in NHEJ repair, with a compensatory increase in TMEJ to nearly $26145 \%$ of all DSB repair (Fig. 5d,e). In contrast, DNA-PK inhibition in POLQ $Q^{-/}$TP53 ${ }^{-/}$RPE1

262 cells did not result in a substantial increase TMEJ signature repair (Fig. 5f,g). However, 
263 a higher proportion of Unclassified repair products were detected (Fig. 5f,g). A limitation

264 of NGS analysis of DSB break repair is that non-amplifiable target loci are not measured.

265 Thus, we used digital PCR to quantify the $L B R$ locus detection rate, relative to a control

266 locus, upon inhibition of DNA-PK and/or Pol $\theta$ (Supplementary Fig. 5g,h). LBR locus

267 detection rates were most reduced upon inhibition of DNA-PK and Pol $\theta$, consistent with

268 overall inhibition of DSB repair (Supplementary Fig. 5h). These observations confirm an

269 essential role for TMEJ in compensatory repair of chromosomal DSBs upon 270 pharmacologic inhibition of DNA-PK.

271 To determine the impact of $P O L Q$ inhibition on cellular viability, we performed 272 clonogenic survival assays in the parental TP53/-- and $P O L Q^{-/-}$TP53/- RPE1 lines treated

273 with NCS with or without DNA-PKi. Genetic deficiency in POLQ resulted in significantly 274 reduced viability after NCS treatment, particularly in combination with DNA-PKi (Fig. 5h).

275 TP53 $^{-/-}$RPE1 cells with inhibition of both TMEJ and NHEJ repair pathways had 276 comparable clonogenic survival to p53-proficient RPE1 cells (see Supplementary Fig.

277 1e). Collectively, these findings indicate that hyperactive end joining repair via NHEJ and 278 TMEJ mediate resistance to DNA damaging therapy induced by p53 deficiency (Fig. 5i).

\section{Discussion}

280 These results recognize enhanced DNA end joining repair capacity as a novel 281 component of therapeutic resistance induced by p53 deficiency, and that loss of functional 282 p53 alone is sufficient to increase hyperactive repair. Our findings indicate that DSB end 283 joining hyperactivity is particularly relevant for suppressing S phase DNA damage burden, 284 which we find is a key determinant of mitotic catastrophe (Fig. 5i). Although NHEJ Is 285 conventionally considered to be most critical for repair in $\mathrm{G} 1$, we observed a relatively 
286 greater impact of DNA-PK inhibition on the fate of S phase cells after treatment with a

287 radiomimetic. There are several potential explanations for this unanticipated observation.

288 First, recent findings suggest that DNA-PK may be dispensable for synapsis formation

289 during NHEJ ${ }^{37}$. Accordingly, repair of "simple" DSBs in G1 phase may have a reduced

290 reliance on DNA-PK, whereas repair of more "complex" DSBs in S phase may require

291 DNA-PK, possibly in partnership with the nuclease Artemis ${ }^{38-40}$. Second, it is possible

292 that DNA-PK inhibition may be more impactful in S phase due to trapping of Ku proteins

293 at DSBs, which inhibits the activation of homologous recombination pathways ${ }^{41}$. Third,

294 DNA-PK may be particularly important in early S phase, when sister chromatids are not

295 broadly present. Notably, we observed a prominent peak of unrepaired DSBs just as

296 p53-deficient cells transitioned from G1 to S phase. Our observation that DSB end joining

297 hyperactivity in p53-deficient cells is highly sensitive to DNA-PK inhibition warrants further

298 mechanistic investigation. Recently, CYREN (cell cycle regulator of NHEJ) has been

299 proposed to be a cell-cycle phase specific inhibitor of the Ku70/80 heterodimer that is

300 critical for restricting NHEJ to $\mathrm{H}^{42}$. It is therefore possible that p53-deficiency may

301 transcriptionally reprogram cell cycle-inhibitors of NHEJ to enable hyperactive repair,

302 though that is beyond the scope of this study.

303 Regulatory mechanisms that confer TMEJ hyperactivity in cancer are not well

304 understood, although transcriptional overexpression of $P O L Q$ has also been observed in

305 breast and ovarian cancers with BRCA1/BRCA2 deficiency or mutations in other genes

306 that confer Pol $\theta$ synthetic lethality ${ }^{33,43}$. Recent work investigating integrated pathway

307 analysis of TP53 deficiency noted POLQ to be frequently overexpressed in TP53 pathway

308 deficient cancers ${ }^{1}$. Our findings, in an isogenic p53-deficient cell line model, indicate that 
309 this relationship may be causal. The mechanism for p53-dependent suppression of POLQ

310 expression remains to be elucidated, and may entail the regulation of non-coding RNAs ${ }^{44}$.

311 The use of TMEJ can also be explained by the potential creation of more complex DSBs

312 upon NHEJ suppression that serve as poor substrates for homologous recombination

313 (HR). Indeed, the molecular mechanisms of NHEJ and TMEJ hyperactivity induced by

314 p53 deficiency warrant further investigation.

315 Radiotherapy and other forms of DNA damaging therapy are employed in the vast

316 majority of cancer patients ${ }^{45}$. Resistance to DNA damaging therapy may thus explain the

317 adverse clinical outcomes associated with TP53 mutations in many different cancer

318 types ${ }^{1}$. Our study supports the investigation of DNA-PK inhibitors administered in

319 combination with DNA damaging therapy (including radiotherapy) in patients with p53-

320 deficient cancers. Additionally, as inhibitors of Pol $\theta$ are currently in development ${ }^{46}$, our

321 study suggests that combined inhibition of both DNA-PK and Pol $\theta$ represents a promising

322 strategy to reverse the therapeutic DNA damage resistance in p53-deficient cancers. 


\section{Materials and Methods}

324 Key Resources Table:

\begin{tabular}{|c|c|c|}
\hline $\begin{array}{l}\text { REAGENT or } \\
\text { RESOURCE }\end{array}$ & SOURCE & IDENTIFIER \\
\hline \multicolumn{3}{|c|}{ Antibodies } \\
\hline $\begin{array}{l}\text { F(ab)2-Goat anti- } \\
\text { Rabbit IgG }(\mathrm{H}+\mathrm{L}) \\
\text { Cross-Adsorbed } \\
\text { Secondary Antibody, } \\
\text { Alexa Fluor } \\
633(1: 10,000 \text { for IF) }\end{array}$ & Thermo Fisher Scientific & $\begin{array}{l}\text { Cat\# A-21072, } \\
\text { RRID:AB_2535733 }\end{array}$ \\
\hline \begin{tabular}{|l|} 
Chicken anti-Mouse \\
IgG $(\mathrm{H}+\mathrm{L})$ Cross- \\
Adsorbed Secondary \\
Antibody, Alexa Fluor \\
$488,(1: 10,000$ for IF) \\
\end{tabular} & Thermo Fisher Scientific & $\begin{array}{l}\text { Cat\# A-21200, } \\
\text { RRID:AB_2535786 }\end{array}$ \\
\hline $\begin{array}{l}\text { Mouse Anti-beta-Actin } \\
\text { Monoclonal Antibody, } \\
\text { Unconjugated, Clone } \\
\text { AC-15 (1:10,000 for } \\
\text { WB) }\end{array}$ & Sigma-Aldrich & Cat\# A1978, RRID:AB_476692 \\
\hline $\begin{array}{l}\text { Rabbit Anti-53BP1 } \\
\text { Polyclonal Antibody } \\
\text { (1:500 for IF) }\end{array}$ & Bethyl & $\begin{array}{l}\text { Cat\# A300-272A, } \\
\text { RRID:AB_185520 }\end{array}$ \\
\hline $\begin{array}{l}\text { Mouse Anti-p53 } \\
\text { (1C12) mAb Antibody ( } \\
1: 1000 \text { for WB) }\end{array}$ & Cell Signaling Technology & Cat\# 2524, RRID:AB_331743 \\
\hline $\begin{array}{l}\text { Rabbit Anti- } \\
\text { phosphorylated } \\
\text { Histone H2AX ( } \gamma- \\
\text { H2AX) Polyclonal } \\
\text { Antibody }(1: 500 \text { for IF) } \\
\end{array}$ & Trevigen & Cat\# 4418-APC-100 \\
\hline \multicolumn{3}{|c|}{$\begin{array}{r}\text { Chemicals, Peptides, and Recombinant Proteins } \\
\end{array}$} \\
\hline Trypsin EDTA & Gibco & $25200-056$ \\
\hline $\begin{array}{l}\text { Polyethylenimine, } \\
\text { Linear (MW 25,000) }\end{array}$ & Polysciences & 23966 \\
\hline $\begin{array}{l}\text { Bovine Serum } \\
\text { Albumin }\end{array}$ & Fisher Scientific & BP9706-160 \\
\hline $\begin{array}{l}\text { Corning }{ }^{\circledR} \text { Cell-Tak }{ }^{\mathrm{TM}} \\
\text { and Tissue Adhesive }\end{array}$ & Corning & 354240 \\
\hline RNAiMax & ThermoFisher & 13778100 \\
\hline \multicolumn{3}{|c|}{ Critical Commercial Assays } \\
\hline PlasmoTest & Invitrogen & REP-PT1 \\
\hline
\end{tabular}




\begin{tabular}{|c|c|c|}
\hline RNAeasy Plus Mini Kit & t Qiagen & 74136 \\
\hline Comet Assay Kit & Trevigen & $4250-050-K$ \\
\hline $\begin{array}{l}\mathrm{Q} 5 \otimes \text { Hot Start High- } \\
\text { Fidelity 2X Master Mix }\end{array}$ & NEB & M0494S \\
\hline $\begin{array}{l}\text { NEBuilder }{ }^{\circledR} \text { HiFi DNA } \\
\text { Assembly Master Mix }\end{array}$ & NEB & E2621L \\
\hline $\begin{array}{l}\text { TOPO } ® \text { TA Cloning } ® \\
\text { Kit for Sequencing }\end{array}$ & Invitrogen & 450030 \\
\hline T4 DNA Ligase & NEB & M0202S \\
\hline EdU-Click 594 & baseclick & BCK-Edu594 \\
\hline $\begin{array}{l}\text { NEON Electroporation } \\
\text { Kit }\end{array}$ & ThermoFisher & MPK1025 \\
\hline \begin{tabular}{|l} 
Cas9 Protein and \\
TracrRNA for Alt-R \\
Electroporation
\end{tabular} & IDT & $\begin{array}{l}\text { Cas9 (s.p. high fidelity) } \\
\# 1081060 \\
\text { TracrRNA } \\
\# 1072532 \\
\end{array}$ \\
\hline & Experimental Models: Cell I & Lines \\
\hline $\begin{array}{l}\text { hTERT-RPE1-Tricolor } \\
\text { Reporter } \\
\text { (PCNA-mCherry, } \\
\text { 53BP1-mVenus, H2B- } \\
\text { mTurquoise) }\end{array}$ & Gift from Dr. Jeremy Purvis & (See Citations) \\
\hline hTERT-RPE1 & ATCC & ATCC $^{\circledR}$ CRL-4000 ${ }^{\text {TM }}$ \\
\hline hTERT-RPE1-TP53-/- & This paper & \\
\hline $\begin{array}{l}\text { hTERT-RPE1-TP53-/- } \\
\text { POLQ-/- }\end{array}$ & This paper & \\
\hline & Oligonucleotides (sgRNAs and & Primers) \\
\hline sgLBR & $\begin{array}{l}\text { GCCGATGGTGAAGTGGTAA } \\
\text { G }\end{array}$ & Synthesized at: IDT \\
\hline sgTP53_Exon2 & TCGACGCTAGGATCTGACTG & IDT \\
\hline $\begin{array}{l}\text { sgTP53_Dwnstream_I } \\
\text { ntron }\end{array}$ & I GAAACTGTGAGTGGATCCAT & IDT \\
\hline sgPOLQ_1 & ACTACTCTCAGCTTGA & IDT \\
\hline sgPOLQ 2 & TCAGGAGCATTGCAGCAGAG & IDT \\
\hline LBR_Fwd & $\begin{array}{l}\text { AAATGGCTGTCTTTCCCAGT } \\
\text { AA }\end{array}$ & EtonBio \\
\hline LBR_Rev & ACGCAGTGGCTAAATCATCC & EtonBio \\
\hline $\begin{array}{l}\text { TP53 RTqPCR Primer } \\
\text { Fwd }\end{array}$ & $\begin{array}{l}\text { GAGGTTGGCTCTGACTGTAC } \\
\mathrm{C}\end{array}$ & EtonBio \\
\hline $\begin{array}{l}\text { TP53 RTqPCR Primer } \\
\text { Rev }\end{array}$ & $\begin{array}{l}\text { TCCGTCCCAGTAGATTACCA } \\
\mathrm{C}\end{array}$ & EtonBio \\
\hline $\begin{array}{l}\text { CDKN1A RTqPCR } \\
\text { Primer Fwd }\end{array}$ & $\begin{array}{l}\text { TCACTGTCTTGTACCCTTGT } \\
\text { GCTT }\end{array}$ & EtonBio \\
\hline $\begin{array}{l}\text { CDKN1A RTqPCR } \\
\text { Primer Rev }\end{array}$ & $\begin{array}{l}\text { AGAAATCTGTCATGCTGGTC } \\
\text { TGCC }\end{array}$ & EtonBio \\
\hline
\end{tabular}




\begin{tabular}{|c|c|c|c|c|}
\hline \multicolumn{2}{|c|}{\begin{tabular}{|l|} 
ONTARGET plus \\
Human TP53 Si-RNA \\
SMARTPOOL
\end{tabular}} & \multicolumn{2}{|c|}{$\begin{array}{l}\text { Horizon Discovery (previously } \\
\text { Dharmacon) }\end{array}$} & L-003329-00-0010 \\
\hline \multicolumn{2}{|c|}{$\begin{array}{l}\text { ONTARGET plus } \\
\text { NON-TARGETTING } \\
\text { control siRNAs } \\
\text { SMARTPOOL }\end{array}$} & \multicolumn{2}{|c|}{$\begin{array}{l}\text { Horizon Discovery (previously } \\
\text { Dharmacon) }\end{array}$} & D-001810-10-05 \\
\hline \multicolumn{2}{|c|}{$\begin{array}{l}\text { ESR1 Genomic Locus } \\
\text { Fwd Primer }\end{array}$} & \multicolumn{2}{|c|}{$\begin{array}{l}\text { ATCTGTACAGCATGAAGTGC } \\
\text { AAGA }\end{array}$} & EtionBio \\
\hline \multirow{2}{*}{\multicolumn{2}{|c|}{$\begin{array}{l}\text { ESR1 Genomic Locus } \\
\text { Rev Primer } \\
\text { ESR1 Genomic Locus } \\
\text { Probe }\end{array}$}} & \multicolumn{2}{|c|}{ CTAGTGGGCGCATGTAGGC } & EtonBio \\
\hline & & \multicolumn{2}{|c|}{$\begin{array}{l}T+C+T+A T+G+A+C C \text { TG } \\
\text { (Locked nucleic acid probe with } \\
\text { HEX) }\end{array}$} & $\begin{array}{l}\text { IDT (LNA : Locked Nucleic Acid } \\
\text { Probe) }\end{array}$ \\
\hline \multicolumn{2}{|c|}{ LBR Locus Probe } & \multicolumn{2}{|c|}{$\begin{array}{l}\text { TGAGATTGAATGTAGCCTTT } \\
\text { CTGGCCCTAA (with FAM) }\end{array}$} & \\
\hline \multicolumn{5}{|c|}{$\begin{array}{l}\text { LBR Nested Sequencing Pri } \\
\text { Purple -> Binds genomic DNA } \\
\text { Green -> Phasing portion of primer } \\
\text { chr1:225423928-225424162 } \\
\text { Size: } 235 \text { base pairs } \\
\text { Forward Primer: } 114 \text { base pairs left of cut } \\
\text { Reverse Primer: } 123 \text { base pairs right of cut } \\
\text { Rcomp = reverse complimentary }\end{array}$} \\
\hline \multicolumn{5}{|c|}{$\begin{array}{l}\text { TCAATTCAAGCTCTGTTCCATCTTTATACTTCACAGTGTAAAGCTGGGAGGTGCTG } \\
\text { TCGTGGCTCAGAATTTCTACTTCATAATAAAGTGAACTCCCAGGCCATCGACCTCT } \\
\text { TACCACTTCACCATCGGCAAATTTCCTACTTGGCATTTTCTATAATTAACCTGAATA } \\
\text { GTTTTAAAGAAAAAATTTGAGTCAATACATACACATTTATGTATTCGTCTTTTTCCA } \\
\text { CAGGCTGA }\end{array}$} \\
\hline $\begin{array}{l}\text { Primer } \\
\text { Name }\end{array}$ & Orientation & Location & & Sequence \\
\hline $\begin{array}{l}\text { LBR2.1 } \\
\text { F0 }\end{array}$ & Forward & \begin{tabular}{|l|} 
chr1:22542392 \\
$8-225423949$
\end{tabular} & $\begin{array}{l}\text { CGACGCTCT } \\
\text { CTGTTCCAT }\end{array}$ & $\begin{array}{l}\text { ITCCGATCTTCAATTCAAGCT } \\
\text { C }\end{array}$ \\
\hline $\begin{array}{l}\text { LBR2.1 } \\
\text { F1 }\end{array}$ & Forward & $\begin{array}{l}\text { chr1:22542392 } \\
7-225423949\end{array}$ & $\begin{array}{l}\text { CGACGCTCT } \\
\text { CTGTTCCAT }\end{array}$ & $\begin{array}{l}\text { TTCCGATCTTTCAATTCAAGCT } \\
\text { C }\end{array}$ \\
\hline $\begin{array}{l}\text { LBR2.1 } \\
\text { F2 }\end{array}$ & Forward & $\begin{array}{l}\text { chr1:22542392 } \\
7-225423949\end{array}$ & $\begin{array}{l}\text { CGACGCTCT } \\
\text { CTCTGTTCC }\end{array}$ & $\begin{array}{l}\text { TTCCGATCTCTTCAATTCAAG } \\
\text { ATC }\end{array}$ \\
\hline $\begin{array}{l}\text { LBR2.1 } \\
\text { F3 }\end{array}$ & Forward & $\begin{array}{l}\text { chr1:22542392 } \\
7-225423949\end{array}$ & $\begin{array}{l}\text { CGACGCTCT } \\
\text { GCTCTGTTC }\end{array}$ & $\begin{array}{l}\text { TTCCGATCTACTTCAATTCAA } \\
\text { SATC }\end{array}$ \\
\hline
\end{tabular}




\begin{tabular}{|c|c|c|c|c|}
\hline $\begin{array}{l}\text { LBR2.1 } \\
\text { F4 }\end{array}$ & Forward & $\begin{array}{l}\text { chr1:22542392 } \\
7-225423949\end{array}$ & \multicolumn{2}{|c|}{$\begin{array}{l}\text { CGACGCTCTTCCGATCTGACTTCAATTCA } \\
\text { AGCTCTGTTCCATC }\end{array}$} \\
\hline $\begin{array}{l}\text { LBR2.1 } \\
\text { F5 }\end{array}$ & Forward & $\begin{array}{l}\text { chr1:22542392 } \\
7-225423949\end{array}$ & \multicolumn{2}{|c|}{$\begin{array}{l}\text { CGACGCTCTTCCGATCTAGACTTCAATTC } \\
\text { AAGCTCTGTTCCATC }\end{array}$} \\
\hline $\begin{array}{l}\text { LBR2.1 } \\
\text { R0 }\end{array}$ & Rcomp & $\begin{array}{l}\text { chr1:22542416 } \\
2-225424143\end{array}$ & \multicolumn{2}{|c|}{$\begin{array}{l}\text { CGTGTGCTCTTCCGATCTTCAGCCTGTGG } \\
\text { AAAAAGACG }\end{array}$} \\
\hline $\begin{array}{l}\text { LBR2.1 } \\
\text { R1 }\end{array}$ & Rcomp & $\begin{array}{l}\text { chr1:22542416 } \\
3-225424143\end{array}$ & \multicolumn{2}{|c|}{$\begin{array}{l}\text { CGTGTGCTCTTCCGATCTATCAGCCTGTG } \\
\text { GAAAAAGACG }\end{array}$} \\
\hline $\begin{array}{l}\text { LBR2.1 } \\
\text { R2 }\end{array}$ & Rcomp & $\begin{array}{l}\text { chr1:22542416 } \\
4-225424143\end{array}$ & \multicolumn{2}{|c|}{$\begin{array}{l}\text { CGTGTGCTCTTCCGATCTGATCAGCCTGT } \\
\text { GGAAAAAGACG }\end{array}$} \\
\hline $\begin{array}{l}\text { LBR2.1 } \\
\text { R3 }\end{array}$ & Rcomp & $\begin{array}{l}\text { chr1:22542416 } \\
5-225424143\end{array}$ & \multicolumn{2}{|c|}{$\begin{array}{l}\text { CGTGTGCTCTTCCGATCTTGATCAGCCTG } \\
\text { TGGAAAAAGACG }\end{array}$} \\
\hline $\begin{array}{l}\text { LBR2.1 } \\
\text { R4 }\end{array}$ & Rcomp & $\begin{array}{l}\text { chr1:22542416 } \\
6-225424143\end{array}$ & \multicolumn{2}{|c|}{$\begin{array}{l}\text { CGTGTGCTCTTCCGATCTCTGATCAGCCT } \\
\text { GTGGAAAAAGACG }\end{array}$} \\
\hline $\begin{array}{l}\text { LBR2.1 } \\
\text { R5 }\end{array}$ & Rcomp & $\begin{array}{l}\text { chr1:22542416 } \\
7-225424143\end{array}$ & \multicolumn{2}{|c|}{ CGTGTGCTCTTCCGATCTAC } \\
\hline \multicolumn{5}{|c|}{ Software and Algorithms } \\
\hline \multicolumn{2}{|c|}{ Python $\geq$ v3.5 } & \multicolumn{2}{|c|}{$\begin{array}{l}\text { G. van Rossum, Python } \\
\text { tutorial, Technical Report CS- } \\
\text { R9526, Centrum voor Wiskund } \\
\text { e en Informatica (CWI), Amster } \\
\text { dam, May } 1995\end{array}$} & https://www.python.org/ \\
\hline \multicolumn{2}{|l|}{ Flow Jo } & \multicolumn{2}{|c|}{$\begin{array}{l}\text { FlowJo }{ }^{\mathrm{TM}} \text { Software (Mac) } \\
\text { [proliferation assay analysis] } \\
\text { Becton, Dickinson and } \\
\text { Company; } 2019 .\end{array}$} & https://www.flowjo.com/ \\
\hline \multicolumn{2}{|c|}{ Graphpad Prism v8 } & \multicolumn{2}{|c|}{ N.A. } & https://www.graphpad.com/ \\
\hline \multicolumn{2}{|c|}{ Fiji } & \multicolumn{2}{|c|}{$\begin{array}{l}\text { Schindelin, J.; Arganda- } \\
\text { Carreras, I. \& Frise, E. et al. } \\
\text { (2012), "Fiji: an open-source } \\
\text { platform for biological-image } \\
\text { analysis", Nature methods } 9(7): \\
676-682, \text { PMID 22743772, } \\
\text { doi:10.1038/nmeth.2019 (on } \\
\text { Google Scholar). }\end{array}$} & $\begin{array}{l}\text { https://imagej.net/Fiji\#Downloa } \\
\text { ds }\end{array}$ \\
\hline \multicolumn{2}{|c|}{ CellProfiler } & \multicolumn{2}{|c|}{$\begin{array}{l}\text { CellProfiler Program Citation: } \\
\text { McQuin C, Goodman A, } \\
\text { Chernyshev V, Kamentsky L, } \\
\text { Cimini BA, Karhohs KW, Doan } \\
\text { M, Ding L, Rafelski SM, } \\
\text { Thirstrup D, Wiegraebe W, } \\
\text { Singh S, Becker T, Caicedo JC, } \\
\text { Carpenter AE (2018). } \\
\text { CellProfiler 3.0: Next- }\end{array}$} & $\begin{array}{l}\text { www.cellprofiler.org } \\
\\
\end{array}$ \\
\hline
\end{tabular}




\begin{tabular}{|c|c|c|}
\hline & $\begin{array}{l}\text { generation image processing } \\
\text { for biology. PloS Biol. } \\
\text { 16(7):e2005970 / doi. PMID: } \\
\text { 29969450 (Research article) } \\
\text { Analyst Software Citation: } \\
\text { Jones TR, Kang IH, Wheeler } \\
\text { DB, Lindquist RA, Papallo A, } \\
\text { Sabatini DM, Golland P, } \\
\text { Carpenter AE (2008) } \\
\text { CellProfiler Analyst: data } \\
\text { exploration and analysis } \\
\text { software for complex image- } \\
\text { based screens. BMC } \\
\text { Bioinformatics 9(1):482/doi: } \\
\text { 10.1186/1471-2105-9-482. } \\
\text { PMID: 19014601 PMCID: } \\
\text { PMC2614436 }\end{array}$ & \\
\hline $\begin{array}{l}\text { NIS Elements AR } \\
\text { software }\end{array}$ & & $\begin{array}{l}\text { https://www.nikon.com/products } \\
\text { Imicroscope- } \\
\text { solutions/lineup/img soft/nis- } \\
\text { elements/ }\end{array}$ \\
\hline $\begin{array}{l}\text { SnapGene software v4 } \\
3.4\end{array}$ & GSL Biotech & https://www.snapgene.com \\
\hline \begin{tabular}{|l} 
Open Comet v1.3.1 \\
\\
\end{tabular} & $\begin{array}{l}\text { BM Gyori, G Venkatachalam, } \\
\text { PS Thiagarajan, D Hsu and MV } \\
\text { Clement. "OpenComet: An } \\
\text { automated tool for comet assay } \\
\text { image analysis", } \\
\text { Redox Biology, 2:457-465, } \\
\text { 2014. }\end{array}$ & http://www.cometbio.org \\
\hline ScarMapper & & $\begin{array}{l}\text { https://github.com/pkMyt1/Scar } \\
\text { Mapper.git }\end{array}$ \\
\hline $\begin{array}{l}\text { Additional Image } \\
\text { Analysis Scripts }\end{array}$ & $\begin{array}{l}\text { Code EV1 } \\
\text { Supplementary and Code } \\
\text { Availability (MATLAB scripts) }\end{array}$ & $\begin{array}{l}\text { PMID: } 30886052 \\
\text { PMID: } 29102360\end{array}$ \\
\hline \multicolumn{3}{|c|}{ Other } \\
\hline Genes & www.Ensembl.org & Ensembl v91 \\
\hline
\end{tabular}

\section{Cell Culture}

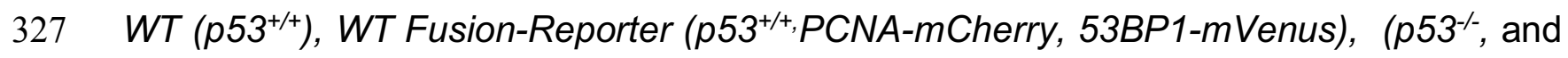
$328 p 53^{--}$Polq ${ }^{-/}$cells are hTERT immortalized RPEs. Cells were maintained in Dulbecco's 
329 modified Eagle's medium (DMEM), with 10\% Fetal Bovine Serum (Hyclone FBS) and

$3302 \mathrm{mM}$ L-glutamine (ThermoFisher). All cells were maintained at $37 \mathrm{C}$ in an atmosphere of

$3315 \%$ CO2. Cells were routinely tested for mycoplasma contamination using PlasmoTest

332 (Invivogen).

333 Establishment of Stable Cell Lines

334 For the TP53 and Polq mutant cell lines, we used the Alt-R-CRISPR-Cas9 system (IDT).

335 We performed Neon transfection (Invitrogen) and followed the manufacturer's protocol

336 with Alt-R HiFi Cas9 nuclease, crRNA and tracrRNA purchased from IDT. crRNA was

337 designed using MIT CRISPR (http://crispr.mit.edu) to target Exon 2 of the TP53 gene for

338 the p53 mutant cell line and the polymerase domain of the Polq gene for the Polq mutant

339 cell line. Forty-eight hours after transfection, cells were seeded for single clone selection.

340 For the p53 gene editing experiment, a homologous template with a stop codon and SCA-

341 I site was provided for selection of gene edited cells. Restriction Enzyme screening, PCR

342 screening, and Sanger sequencing confirmed gene targeting, post which we performed

343 functional tests.

344 Immunofluorescence

345 Cells were fixed with 3\% Paraformaldehyde for 15 min at RT, followed by permeabilization

346 with $0.25 \%$ TritonX-100 in PBS. Cells were subsequently processed for immunostaining

347 experiments using the antibodies listed below. Nuclei were visualized by staining with

348 DAPI. The primary antibodies used were: $y H 2 A X(1: 500$, Trevigen, 4418-APC-100), and

349 53BP1 (1:500 for immunofluorescence, Bethyl, A300-272A). The secondary antibodies

350 were: FITC Goat Anti-Mouse IgG $(H+L)$ (1:500, Jackson ImmunoResearch, 115-095-

351 003) and FITC Goat Anti Rabbit IgG $(H+L)(1: 500$, Jackson ImmunoResearch, 111-095- 
144). Images were acquired using the GE IN CELL 2200 high through-put imaging system

353 at $40 x$ magnification.

354 siRNA Treatment

355 WT Fusion-Reporter RPE cells were passaged twice after -80 thaw and plated on 12 well

356 plates at a density of 100,000 cells/ well for siRNA treatment. Twenty-four hours post

357 plating, cells were exposed to 10nM / well sip53 (SMART pool from Dharmacon), and

358 siControl (Non-targetting SMART pool from Dharmacon), in OPTIMEM with RNA-iMAX

359 (ThermoFisher) as a transfection reagent. As a no-treatment control, cells were exposed

360 to RNA-iMAX and OPTIMEM without siRNA. 48 hours post transfection, cells were

361 transferred onto 12 well Cell-Tak coated glass plates (Cellvis), at a concentration of

36250,000 cells/well for imaging. Prior to imaging and at the end of imaging, samples were

363 taken for RT-qPCR analysis of p53 mRNA to confirm si-RNA knockdown.

364 Mixed Competition Assay - Flow Cytometry

365 mCherry labelled and unlabeled hTERT-RPE1 cell lines were plated on 96 well plates at

366 a 50:50 ratio, and irradiated 2 hrs post plating at 0,2, 4, or $6 \mathrm{~Gy}$, and left to grow. At

367 indicated timepoints cells were harvested by trypsinizing and quenching with PBS with

$3685 \%$ BSA. Cells were fixed with $2 \%$ PFA and subsequently transferred to V-bottom plates

369 (ThermoFisher, 249570). Cells were quantified by flow cytometry using the Intellicyt iQue

370 at a volume of 100 ul / sample, collecting all events per well. For each condition, 6

371 biological replicates.

\section{$372 \quad$ Time-Lapse Imaging Microscopy}

373 Cells stably expressing Proliferating Cell Nuclear Antigen (PCNA)-mCherry and Tumor 374 Suppressor p53 Binding Protein 1 (53BP1) - mVenus were treated with si-RNA for 48 
375 hours prior to imaging. PCNA-mCherry and 53BP1-mVenus fusion reporter is a gift from

376 Dr. Jeremy Purvis and Hui Chao Xiao. Cells were plated on Cell-Tak (Corning) coated

377 glass-bottom 12-well plates (Cellvis) with Phenol-free DMEM (Invitrogen) supplemented

378 with 10\% FBS, and L-glutamine. Twenty-four hours post plating, cells were image

379 captured every $10 \mathrm{~min}$ for $72 \mathrm{~h}$ in the mCherry and mVenus fluorescence channels. 18

380 hours into imaging, DNA PKi was added at a concentration of $0.5 \mathrm{uM} /$ well, and/or NCS

381 at a concentration of $100 \mathrm{ng} / \mathrm{mL} /$ well. We commenced imaging every 10 minutes in both

382 channels for another 48 hours. Fluorescence images were obtained using a Nikon Ti

383 Eclipse inverted microscope with a 40x objective and Nikon Perfect Focus (PFS) system

384 to maintain focus during acquisition period. Cells were maintained at constant

385 temperature $\left(37^{\circ} \mathrm{C}\right)$ and atmosphere $\left(5 \% \mathrm{CO}_{2}\right)$. Nikon, NIS Elements AR software was

386 utilized for image acquisition. Image analysis was performed on ImageJ - Fiji and Cell

387 Profiler.

388 Colony Forming Assays

389 Cells lines used in the assay are indicated in the figures. Cells were treated with NCS

390 and/ or DNA-PKi for twenty-four hours, after which we performed a media change. For

391 colony formation experiments with ionizing radiation, cells were plated for IR treatment

392 with or without DNA-PKi, and inhibitor treatment was washed off after twenty-four hours.

393 Cells were subsequently incubated for $10-12$ days at $37^{\circ} \mathrm{C}$ to allow colony formation.

394 Colonies were stained by Coomassie blue and counted.

\section{DNA Repair Assay}

396 Cell lines used in the assay are indicated in the figure. $5 \times 10^{5}$ cells were transfected with

397 sgLBR2 and TracrRNA complexed Cas9 protein at final concentrations of 
sgRNA:tracrRNA duplex: 22 pmol and Cas9 : 18 pmol per reaction, with Neon transfection

399 kit (Invitrogen) using $21350 \mathrm{~V}, 30 \mathrm{~ms}$ pulses in a $10 \mu \mathrm{L}$ chamber. 60 hours post

400 transfection, cells were harvested for genomic DNA extraction (Nucleospin). Part of the

401 gDNA was utilized for Sanger Sequencing and TIDE analysis post amplification of the

402 genomic LBR2 locus. Remaining gDNA was amplified using NGS nested sequencing

403 primers and sent for sequencing and/ or Digital PCR.

404 Digital PCR

405 Primers and 5' hydrolysis probes were designed to specifically detect the copies of $L B R$ 406 locus. ESR1 locus was used as genomic control. Each reaction assay contained $10 \mu$ Lof $4072 x$ dPCR Supermix for Probes (No dUTP), $0.9 \mu \mathrm{mol} / \mathrm{L}$ of respective primers, $0.25 \mu \mathrm{mol} / \mathrm{L}$ 408 of respective probes, and $10 \mathrm{ng}$ of DNA in a final volume of $20 \mu \mathrm{L}$. Droplets were 409 generated using automated droplet generator (Bio-Rad catalog \#186-4101) following 410 manufacturer's protocol. PCR parameters for $L B R$ locus were $10 \mathrm{sec}$ at $95^{\circ} \mathrm{C}$, then 40 411 cycles of $94{ }^{\circ} \mathrm{C}$ for $30 \mathrm{sec}, 60^{\circ} \mathrm{C}$ for $30 \mathrm{sec}$, and $72^{\circ} \mathrm{C}$ for $2 \mathrm{~min}$ followed by $98^{\circ} \mathrm{C}$ for 10 412 min with a ramping of $2{ }^{\circ} \mathrm{C} / \mathrm{sec}$ at all steps. The PCR cycling parameters for ESR1 413 genomic locus were $10 \mathrm{sec}$ at $95^{\circ} \mathrm{C}$, then 40 cycles of $94^{\circ} \mathrm{C}$ for $30 \mathrm{sec}$ and $60{ }^{\circ} \mathrm{C}$ for 1 414 min followed by $98^{\circ} \mathrm{C}$ for 10 min with a ramping of $2{ }^{\circ} \mathrm{C} / \mathrm{sec}$ at all steps. After PCR 415 amplification, droplet reader (Bio-Rad QX200 ${ }^{\text {TM }}$ Droplet Reader Catalog \#1864003) was 416 used to measure the end-point fluorescence signal in droplets as per the manufacturer's 417 protocol. The recorded data was subsequently analyzed with QuantaSoft software 418 version 1.7.4.0917 (Bio-Rad). Each Taqman probe was evaluated for sensitivity and 419 specificity.

420 DNA Repair Assay 
421 Cell lines used in the assay are indicated in the figure. $5 \times 10^{5}$ cells were transfected with

422 sgLBR2 and TracrRNA complexed Cas9 protein at final concentrations of

423 sgRNA:tracrRNA duplex: 22 pmol and Cas9 : 18 pmol per reaction, with Neon transfection

424 kit (Invitrogen) using $21350 \mathrm{~V}, 30 \mathrm{~ms}$ pulses in a $10 \mu \mathrm{L}$ chamber. 60 hours post

425 transfection, cells were harvested for genomic DNA extraction (Nucleospin). Part of the

426 gDNA was utilized for Sanger Sequencing and TIDE analysis post amplification of the

427 genomic LBR2 locus. For analysis of INDELs, 100 ng of gDNA was amplified using

428 phased primers. These libraries were indexed with the Illumina unique dual combinatorial

429 indices. Following pooling, 2 × 150 cycle sequencing was done on an Illumina iSeq $^{\mathrm{TM}}$.

430 INDELs were identified by comparing the target reference sequence to the resulting

431 sequence reads in the FASTQ files via a 10-nucleotide sliding window using the

432 ScarMapper program.

433

434 Acknowledgements

435 We thank Kasia Kedziora, Samuel Wolff, and Juan Carvajal-Garcia for data acquisition

436 and technical assistance. We are grateful to the Gupta and Purvis Lab members for

437 helpful discussions. UNC Core labs (Microscopy Services Laboratory, Hooker Imaging

438 Core, and Flow Cytometry Core Facility) used in this study are supported in part by P30

439 CA016086 Cancer Center Core Support Grant to the UNC Lineberger Comprehensive

440 Cancer Center. Funding support was provided by the NCI/NIH (CA222092), Dept of

441 Defense (W81XWH-18-1-0047), and the University Cancer Research Fund. G.P.G. holds

442 a Career Award for Medical Scientists from the Burroughs Wellcome Fund. R.J.K. is 
443 supported by the Cancer Cell Biology T32 Training Program (2T32CA071341-21) and the

444 UNC Medical Scientist Training Program (MSTP).

\section{Author contributions}

447 R.J.K and G.P.G. designed and conceived experiments. G.P.G. supervised the study.

448 R.J.K and H.C.X performed the live-cell imaging experiments. H.C.X. and J.E.P. provided 449 critical reagents and image analysis guidance. R.J.K performed and implemented 450 computational analyses for image processing. R.J.K. performed all additional 451 experiments and data analyses with statistical review. V.R.R., A.R.S., S.J.S., W.F., A-

452 S.W., and S.K. provided technical assistance on imaging acquisition, colony forming 453 assays, and digital PCR. D.A.S. developed break site sequencing analysis platforms. 454 R.J.K. and G.P.G. wrote the manuscript, with contributions from all authors. All authors 455 read and accepted the manuscript.

\section{Conflicts of interests}

458 The authors declare no competing interests that are pertinent to this study.

460 Correspondence

461 Correspondence should be addressed to Gaorav P. Gupta: gaorav_gupta@med.unc.edu

\section{References}

463 1. Donehower, L. A. et al. Integrated Analysis of TP53 Gene and Pathway

$464 \quad$ Alterations in The Cancer Genome Atlas. Cell Rep. 28, 1370-1384.e5 (2019).

465 2. Lowe, S. W. et al. p53 status and the efficacy of cancer therapy in vivo. Science 
(80-. ). 266, 807-810 (1994).

3. Lee, J. M. \& Bernstein, A. p53 Mutations increase resistance to ionizing radiation.

4. Wyatt, D. W. et al. Essential Roles for Polymerase $\theta$-Mediated End Joining in the

5. Nigro, J., Baker, S., Preisinger, A., Nature, J. J.- \& 1989, undefined. Mutations in the p53 gene occur in diverse human tumour types. Springer (1989).

473

475

6. Hafner, A., Bulyk, M. L., Jambhekar, A. \& Lahav, G. The multiple mechanisms that regulate p53 activity and cell fate. Nature Reviews Molecular Cell Biology 20, 199-210 (2019).

7. Kastenhuber, E. R. \& Lowe, S. W. Leading Edge Putting p53 in Context. (2017). doi:10.1016/j.cell.2017.08.028

8. Young, K. H. et al. Structural profiles of TP53 gene mutations predict clinical outcome in diffuse large B-cell lymphoma: An international collaborative study. Blood 112, 3088-3098 (2008).

9. Miller, L. D. et al. An expression signature for p53 status in human breast cancer predicts mutation status, transcriptional effects, and patient survival. Proc. Natl. Acad. Sci. U. S. A. 102, 13550-13555 (2005).

10. Robles, A. I. \& Harris, C. C. Clinical outcomes and correlates of TP53 mutations and cancer. Cold Spring Harbor perspectives in biology 2, (2010).

11. Freed-Pastor, W. A. \& Prives, C. Mutant p53: one name, many proteins. genesdev.cshlp.org doi:10.1101/gad.190678.112 
12. Lee, M. K. et al. Cell-type, Dose, and Mutation-type Specificity Dictate Mutant p53 Functions In Vivo. Cancer Cell 22, 751-764 (2012).

13. Bykov, V. J. N. et al. Restoration of the tumor suppressor function to mutant p53 by a low-molecular-weight compound. Nat. Med. 8, 282-288 (2002).

14. Huang, S. et al. P53 modulates acquired resistance to EGFR inhibitors and radiation. Cancer Res. 71, 7071-7079 (2011).

15. Aubrey, B. J., Kelly, G. L., Janic, A., Herold, M. J. \& Strasser, A. How does p53 induce apoptosis and how does this relate to p53-mediated tumour suppression? Cell Death and Differentiation 25, 104-113 (2018).

16. Nardella, C., Clohessy, J. G., Alimonti, A. \& Pandolfi, P. P. Pro-senescence therapy for cancer treatment. Nature Reviews Cancer 11, 503-511 (2011).

17. Vitale, I., Galluzzi, L., Castedo, M. \& Kroemer, G. Mitotic catastrophe: a mechanism for avoiding genomic instability. Nat. Rev. Mol. Cell Biol. 12, 385-392 (2011).

18. Williams, A. B. \& Rn Schumacher, B. p53 in the DNA-Damage-Repair Process. perspectivesinmedicine.cshlp.org doi:10.1101/cshperspect.a026070

19. Reyes, J. et al. Fluctuations in p53 Signaling Allow Escape from Cell-Cycle Arrest. Mol. Cell 71, 581-591.e5 (2018).

20. Zhang, X. P., Liu, F., Cheng, Z. \& Wang, W. Cell fate decision mediated by p53 pulses. Proc. Natl. Acad. Sci. U. S. A. 106, 12245-12250 (2009). 
Reviews Molecular Cell Biology 18, 495-506 (2017).

$51122 . \quad$ Krenning, L., Feringa, F. M., Shaltiel, I. A., vandenBerg, J. \& Medema, R. H.

512 Transient activation of p53 in G2 phase is sufficient to induce senescence. Mol.

$513 \quad$ Cell 55, 59-72 (2014).

514 23. Chao, H. X. et al. Evidence that the human cell cycle is a series of uncoupled, 515 memoryless phases. Mol. Syst. Biol. 15, (2019).

516 24. Chao, H. X. et al. Orchestration of DNA Damage Checkpoint Dynamics across the $517 \quad$ Human Cell Cycle. Cell Syst. 5, 445-459.e5 (2017).

518 25. Chao, H. X. et al. Orchestration of DNA Damage Checkpoint Dynamics across the Human Cell Cycle. Cell Syst. 0, (2017).

520 26. Chao, H. X. et al. Evidence that the cell cycle is a series of uncoupled, memoryless phases. (2018). doi:10.1101/283614

522 27. Blackford, A. N. \& Jackson, S. P. Molecular Cell Review ATM, ATR, and DNA-PK:

523 The Trinity at the Heart of the DNA Damage Response. (2017).

524 doi:10.1016/j.molcel.2017.05.015

525 28. Zhao, Y. et al. Preclinical evaluation of a potent novel DNA-dependent protein

$526 \quad$ kinase inhibitor NU7441. Cancer Res. 66, 5354-5362 (2006).

527 29. Chan, D. W. et al. Autophosphorylation of the DNA-dependent protein kinase 528 catalytic subunit is required for rejoining of DNA double-strand breaks. Genes

$529 \quad$ Dev. 16, 2333-2338 (2002).

530 30. Attardi, L. D., De Vries, A. \& Jacks, T. Activation of the p53-dependent G1 531 checkpoint response in mouse embryo fibroblasts depends on the specific DNA 
damage inducer. Oncogene 23, 973-980 (2004).

533 31. Agarwal, M. L., Agarwal, A., Taylor, W. R. \& Stark, G. R. p53 controls both the G2/M and the G1 cell cycle checkpoints and mediates reversible growth arrest in human fibroblasts. Proc. Natl. Acad. Sci. U. S. A. 92, 8493-8497 (1995).

32. Pawlik, T. M. \& Keyomarsi, K. Role of cell cycle in mediating sensitivity to radiotherapy. Int. J. Radiat. Oncol. 59, 928-942 (2004).

33. Feng, W. et al. Genetic determinants of cellular addiction to DNA polymerase theta. Nat. Commun. 10, 1-13 (2019).

34. Wood, R. D. \& Doublié, S. DNA polymerase $\theta$ (POLQ), double-strand break repair, and cancer. DNA Repair 44, 22-32 (2016).

35. Brinkman, E. K. et al. Kinetics and Fidelity of the Repair of Cas9-Induced DoubleStrand DNA Breaks Molecular Cell Article Kinetics and Fidelity of the Repair of Cas9-Induced Double-Strand DNA Breaks. Mol. Cell 70, 801-813 (2018).

545 36. Brinkman, E. K., Chen, T., Amendola, M. \& Van Steensel, B. Easy quantitative assessment of genome editing by sequence trace decomposition. Nucleic Acids

37. Zhao, B. et al. The essential elements for the noncovalent association of two DNA ends during NHEJ synapsis. Nat. Commun. 10, (2019).

38. Bétous, R. et al. DNA replication stress triggers Rapid DNA Replication Fork Breakage by Artemis and XPF. PLoS Genet. 14, (2018).

552 39. Ma, Y., Pannicke, U., Schwarz, K. \& Lieber, M. R. Hairpin opening and overhang processing by an Artemis/DNA-dependent protein kinase complex in 
nonhomologous end joining and V(D)J recombination. Cell 108, 781-794 (2002).

555 40. Löbrich, M. \& Jeggo, P. A Process of Resection-Dependent Nonhomologous End Joining Involving the Goddess Artemis. Trends in Biochemical Sciences 42, 690_

41. Zhou, Y. \& Paull, T. T. DNA-dependent protein kinase regulates DNA end resection in concert with Mre11-Rad50-Nbs1 (MRN) and Ataxia Telangiectasiamutated (ATM). J. Biol. Chem. 288, 37112-37125 (2013).

42. Arnoult, N. et al. Regulation of DNA repair pathway choice in S and G2 phases by the NHEJ inhibitor CYREN. Nature 549, 548-552 (2017).

43. Ceccaldi, R. et al. Homologous recombination-deficient tumors are hyperdependent on POLQ-mediated repair. Nature 518, 258-262 (2015).

44. Huarte, M. et al. A large intergenic noncoding RNA induced by p53 mediates global gene repression in the p53 response. Cell 142, 409-419 (2010).

45. Baskar, R., Dai, J., Wenlong, N., Yeo, R. \& Yeoh, K. W. Biological response of cancer cells to radiation treatment. Frontiers in Molecular Biosciences 1, 24 (2014).

46. Higgins, G. S. \& Boulton, S. J. Beyond PARP-POL $\theta$ as an anticancer target. 
a
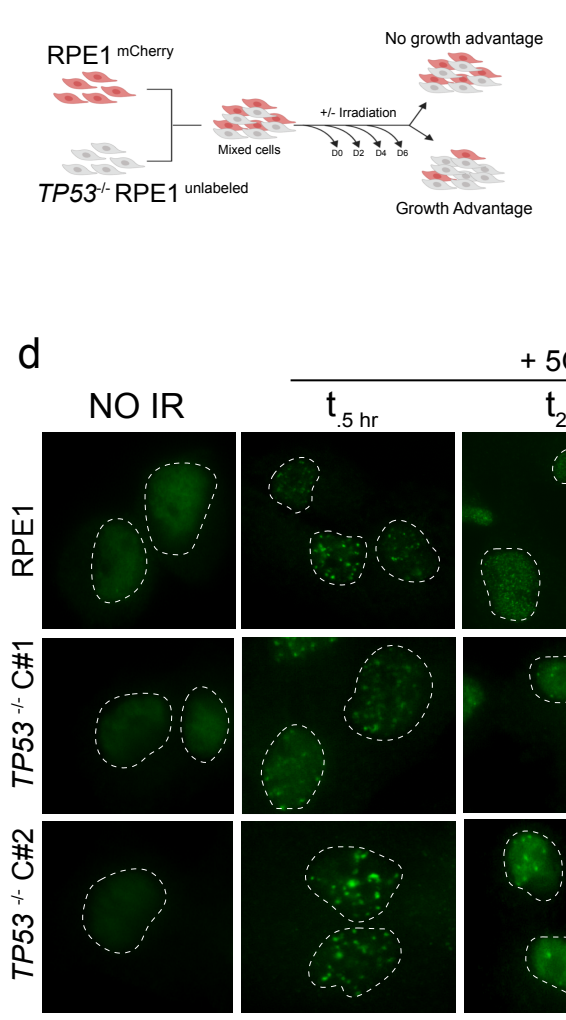

f

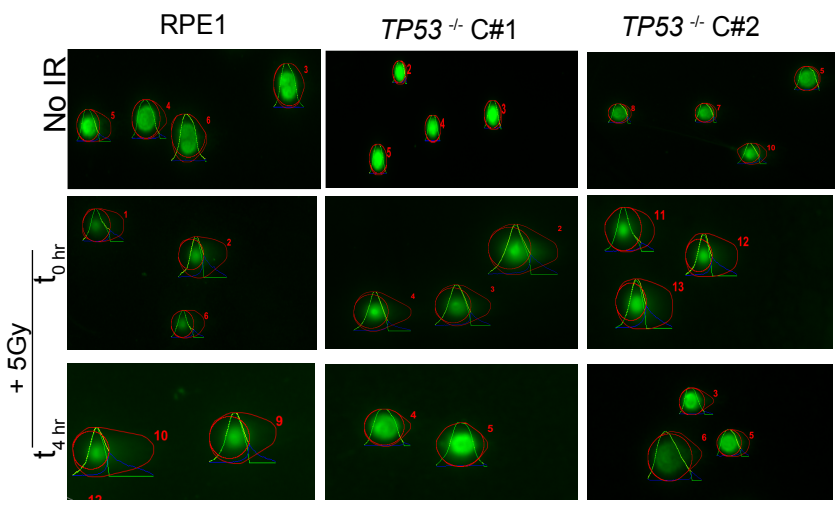

Figure 1

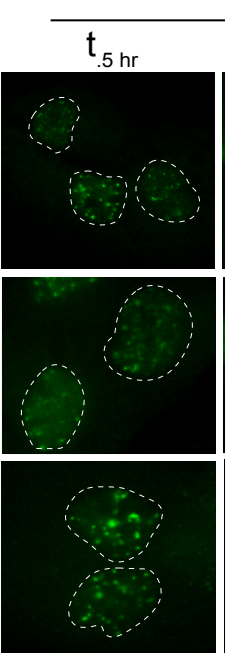

$+5 \mathrm{G}$

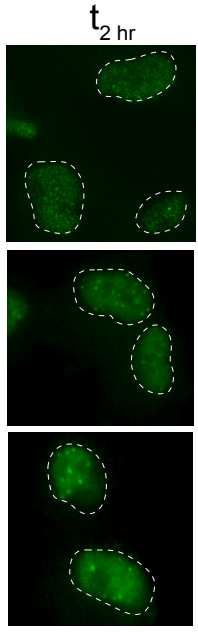

b

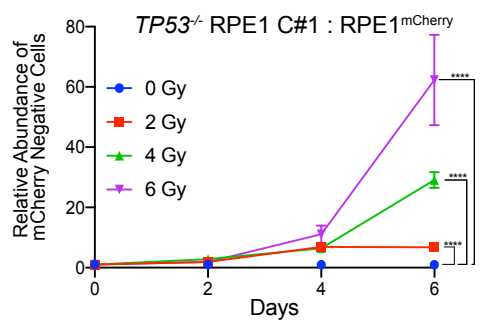

C

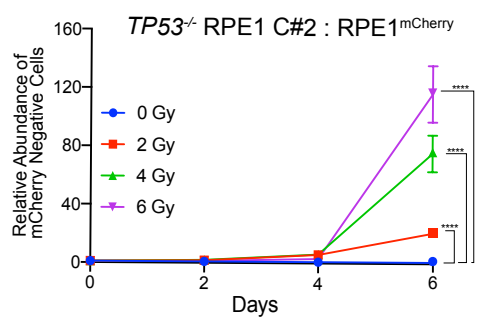

e
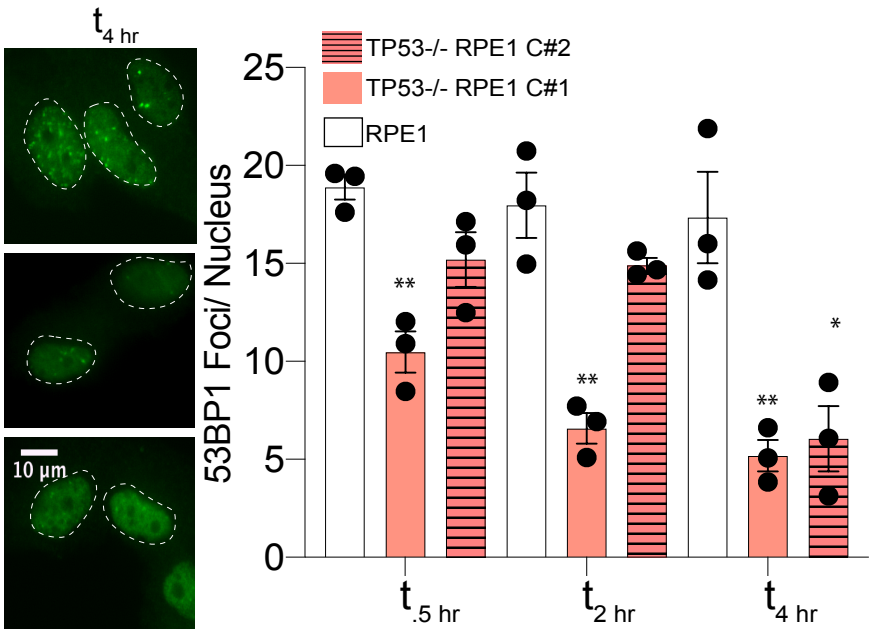

g

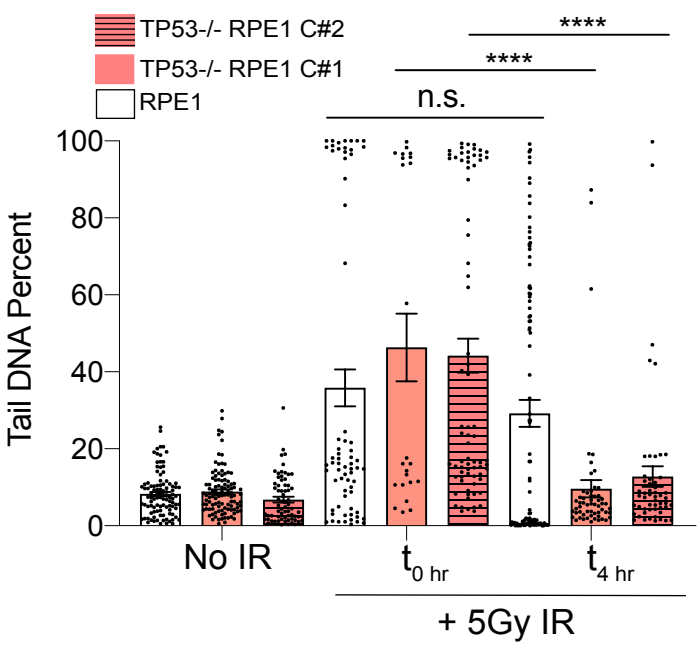


Figure 1 | p53-deficient cells exhibit radioresistance and accelerated resolution of DNA DSBs.

a, Diagram of growth competition assay. mCherry-labelled RPE1 cells were mixed with unlabeled TP53 $3^{-/-}$RPE1 (1:1), exposed to IR and grown for 6 days. b, Relative abundance of unlabeled TP5 $3^{-/}$Clone\#1 measured by Intellicyte high-throughput cytometry \pm SEM $(n=6)$ is shown, normalized to the untreated (0Gy) cohort at each time point. c, Relative abundance of unlabeled TP53 $\%$ Clone\#2 \pm SEM $(n=6)$ is shown, normalized to the untreated (0Gy) cohort at each time point. d, Representative immunofluorescence images of 53BP1 foci in cells with indicated genotypes untreated (no IR) or treated with IR (5Gy) and collected at .5, 2, and $4 \mathrm{~h}$ after irradiation. e, Quantification of 53BP1 foci. Data shown are mean ( $n=50$ cells per treatment condition) \pm SEM $(n=3)$, and are consistent across two independent biological replicates. ${ }^{*} p<0.05$; ${ }^{* *} p<0.01$; by twotailed Student's t-test. f, Representative Neutral COMET fluorescence staining for DNA tails in cells with indicated genotypes treated with or without 5Gy IR. For irradiated cells, 2 timepoints are shown: immediately after and 4 hours post IR. COMET tails and heads are denoted by OpenComet software analysis. g, Quantification of DNA DSBs via Neutral COMET assay reported as tail DNA percent at 0 and 4 hours post IR in RPE1 and two TP53-- RPE1 cell lines. Data shown are mean ( $n=50-150$ cells per treatment condition) \pm SEM, and are consistent across three independent biological replicates. ${ }^{*} p<0.05$; ${ }^{* *} p<0.01 ;{ }^{* * *} p<0.0001$ by two-tailed Student's t-test. 


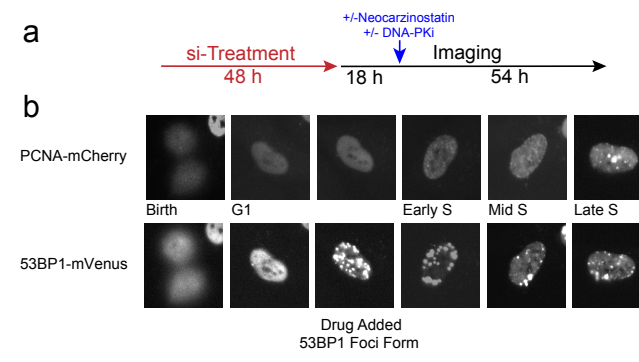

d

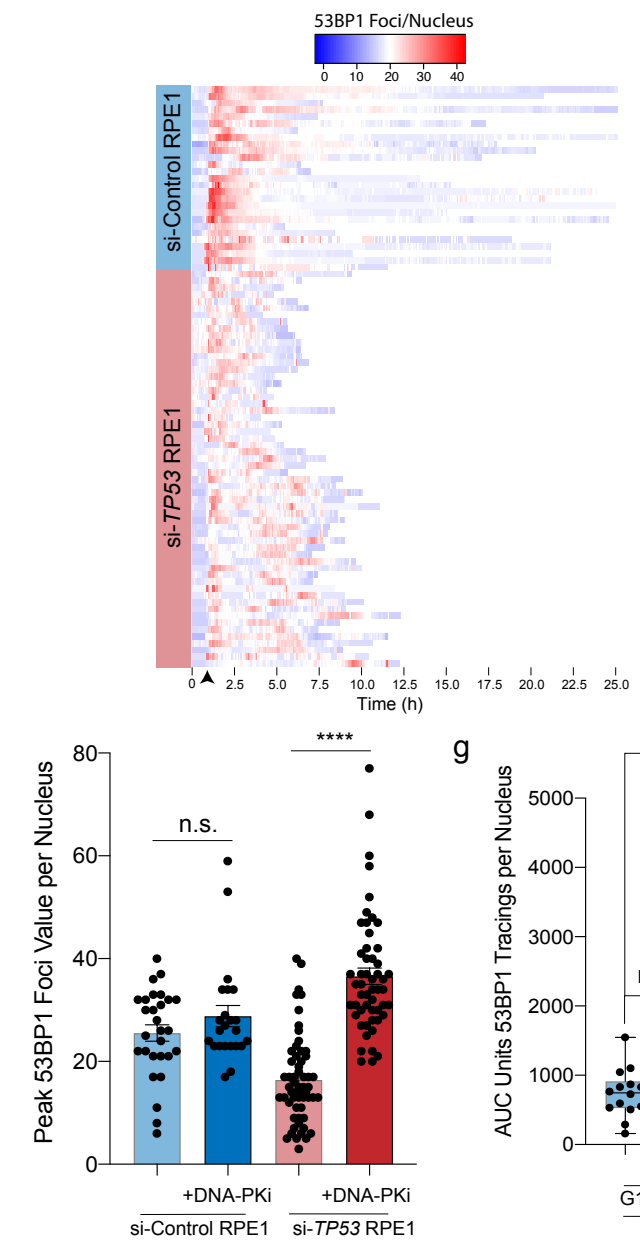

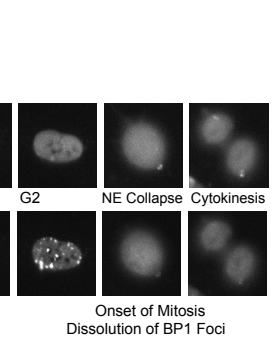

e
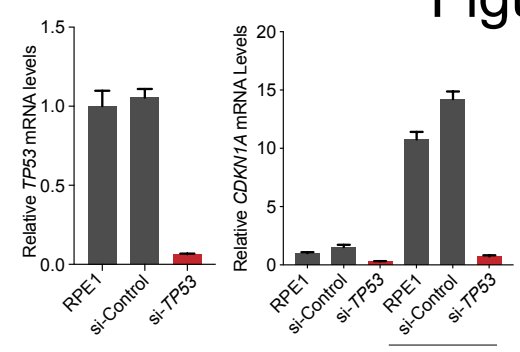

Figure 2

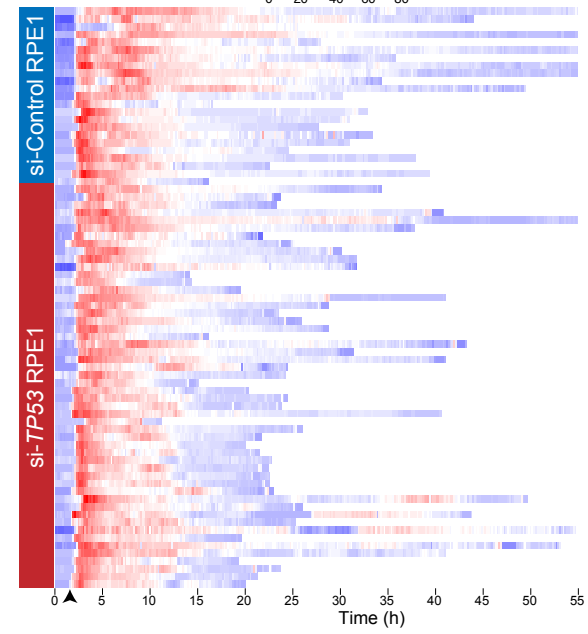

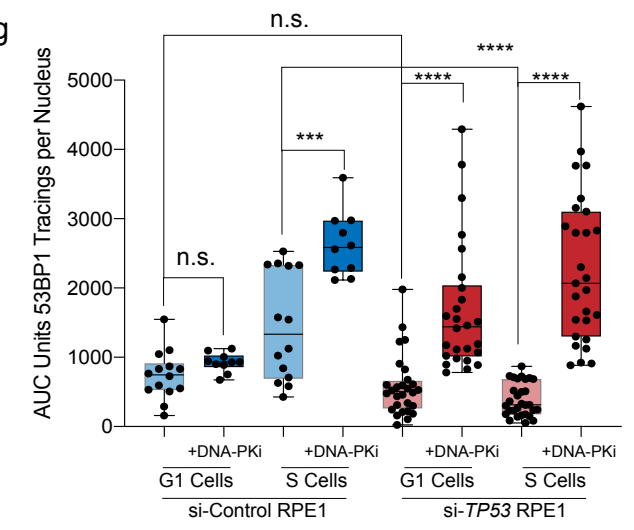

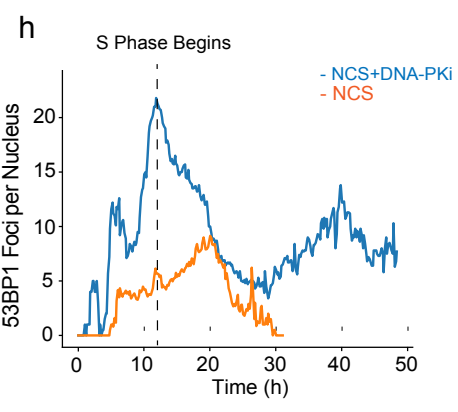


Figure 2 | Inhibition of DNA-PK restores DNA damage foci formation in p53deficient cells.

a, Live cell imaging procedure. Cells transfected with $10 \mathrm{nM}$ si-control or si-TP53 for 48 $\mathrm{h}$ prior to imaging. $18 \mathrm{~h}$ into imaging, cells are treated with NCS (100 nM), DNA-PKi (.5 $\mathrm{uM}$ ) or both and imaged for 72 total hours. b, RPE1 cell expressing the PCNA-mCherry and 53BP1-mVenus reporters. Cell cycle phases delineated by PCNA foci and DNA DSBs are marked by 53BP1 foci. c, RT-qPCR for TP53 mRNA levels (left) and CDKN1A mRNA levels (right) in si-control treated vs. si-TP53 treated cells. To induce CDKN1A expression, cells irradiated at 5Gy and mRNA harvested 3 hrs post IR. d, Heatmap of 53BP1 foci tracings for single cells tracked from birth to mitosis or end of imaging. For sicontrol ( $n=30$ cells) and si-TP53 treated RPE1 $(n=60$ cells) treated with NCS $100 \mathrm{ng} / \mathrm{ml}$. For visualization, cells are aligned to 10 frames prior to drug addition (black arrow). e, Heatmap of 53BP1 foci tracings for si-control ( $n=25$ cells) and si-TP53 treated RPE1 cells $(n=55$ cells) treated with $100 \mathrm{ng} / \mathrm{ml}$ NCS +0.5 uM DNA-PKi. f, Peak 53BP1 foci counts for cells treated with $100 \mathrm{ng} / \mathrm{ml}$ NCS or NCS+0.5 uM DNA-PKi. Significance determined using two-tailed t-test. g, Area under the curve (AUC) analysis of 53BP1 burden showing integral DNA damage for cells treated with NCS vs. NCS and DNA-PKi. Cells are segregated into two groups: cells exposed to drug in G1 vs. S phase ( $n=25-$ 30 G1or $S$ cells for si-TP53 cohort, $n=10-15$ G1 or $S$ cells for si-control cohort). Significance determined by two-tailed t-test. ${ }^{* * * *} p<0.0001,{ }^{* * *} p<0.001$, n.s. $=$ nonsignificant. $\mathbf{h}, 53 \mathrm{BP} 1$ foci burden in G1 vs. S phase p53-deficient RPE1 upon exposure to NCS and DNA-PKi. Dashed line $=\mathrm{S}$ phase onset, blue line $=$ mean 53BP1 foci burden for all cells in G1 with NCS and DNA-PKi addition, orange line $=$ mean foci value for cells in $\mathrm{G} 1$ with NCS treatment alone, ( $n=30$ cells for each condition). 
Figure 3

a

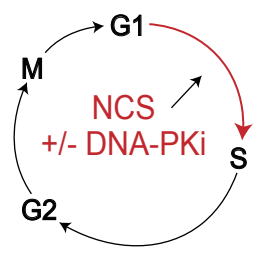

C

e

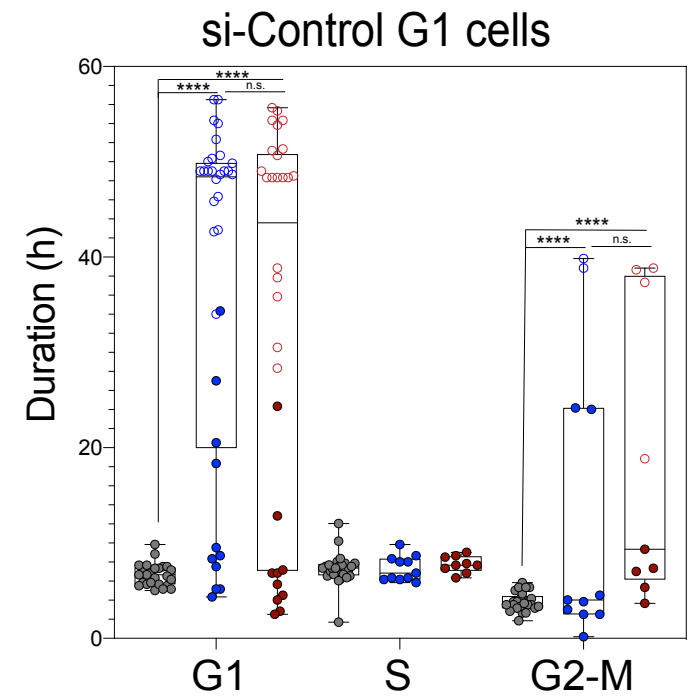

b

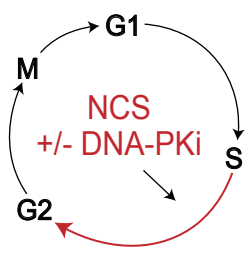

d
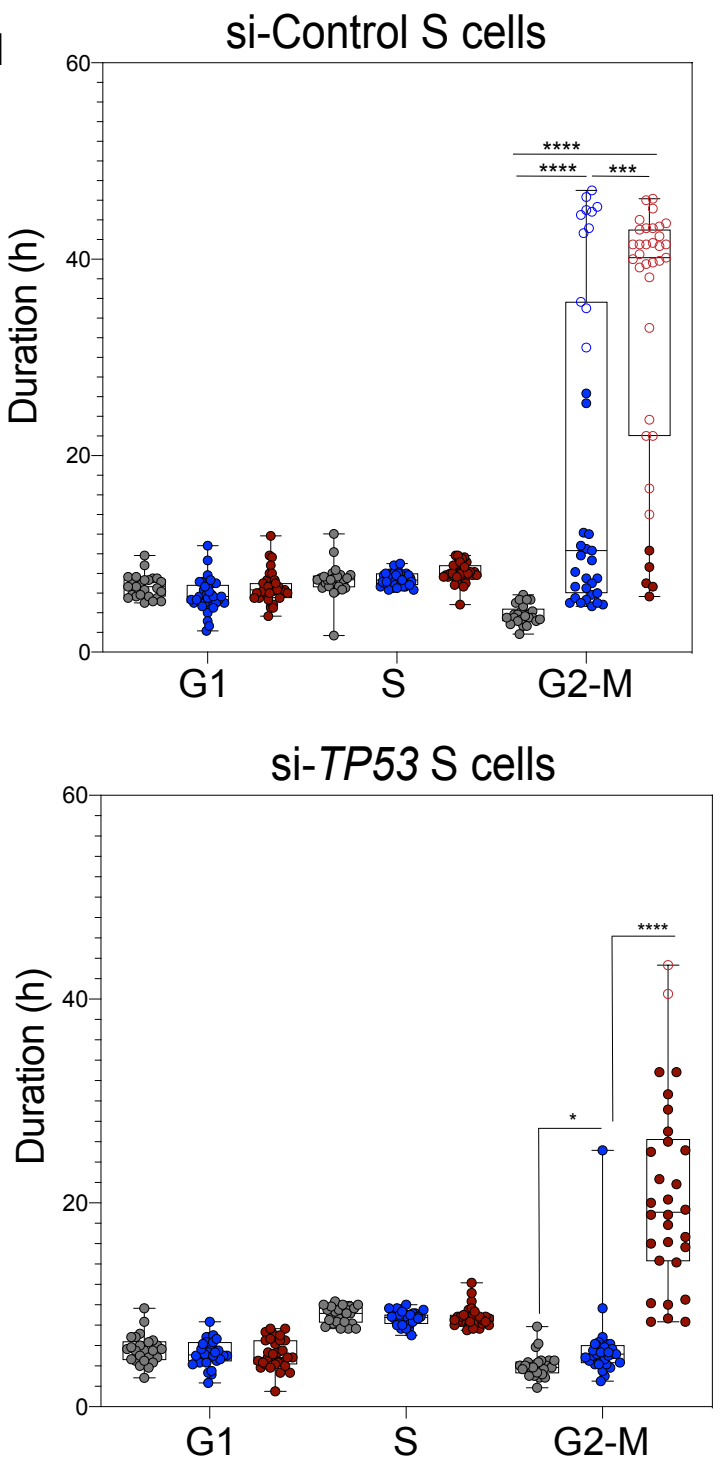
Figure 3 | Checkpoint responses halt p53-proficient cells upon exposure to NCS while p53-deficient cells continue to cell cycle despite NCS exposure.

a, Schematic depicting NCS treatment $(50 \mathrm{ng} / \mathrm{ml}+100 \mathrm{ng} / \mathrm{ml}$ for si-control and $100 \mathrm{ng} / \mathrm{ml}$ for si-TP53 RPE1) and/or NCS + 0.5 uM DNA-PKi treatment, and phase of the cell cycle cells are exposed to drug (G1). b, Schematic of drug treatment for $S$ phase cells. c, Distribution of cell cycle phase lengths, each colored dot is an individual cell with untreated cells (no NCS) shown in black, NCS treated cells shown in blue, and NCS+ 0.5uM DNA-PKi treated cells shown in red for si-control RPE1 in G1 phase. $n=20$ untreated and $n=30$ treated cells (for each treatment cohort). Statistical significance was determined by comparing untreated and treated groups at each phase. ${ }^{* * * *} p<0.0001$, n.s. = non-significant. Open circles indicate arrested cells that did not enter the subsequent phase of cell cycle for remainder of imaging. $\mathbf{d}$, Distribution of cell cycle phase lengths for si-control treated RPE1 in S phase, ${ }^{* *} p<0.001,{ }^{* * * *} p<0.0001$, n.s. $=$ nonsignificant as evaluated by two-tailed t-test. e, Distribution of cell cycle phase lengths for si-TP53 treated RPE1 in G1 phase, ${ }^{* * * *} p<0.0001$, n.s. = non-significant as evaluated by two-tailed t-test. $\mathbf{f}$, Distribution of cell cycle phase lengths for si-TP53 treated RPE1 in S phase, ${ }^{* * * *} p<0.0001$, n.s. $=$ non-significant as evaluated by two-tailed t-test. 
a
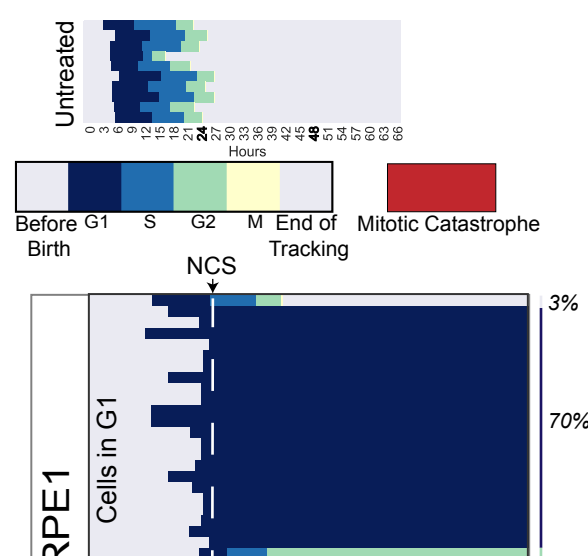

$70 \%$

$\frac{n}{\alpha}$

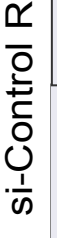

$\frac{1}{\infty} \cos$
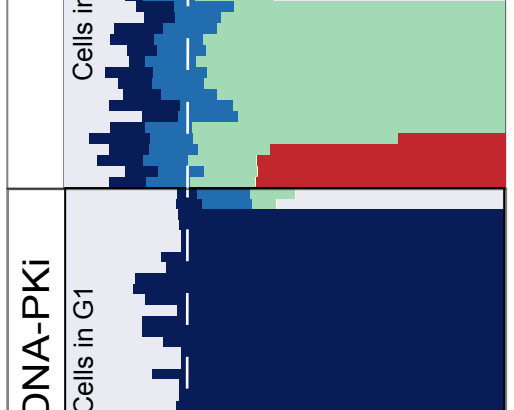

$+$

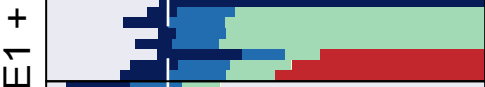

믐

은

을

$-\frac{1}{\omega}$

0 ;

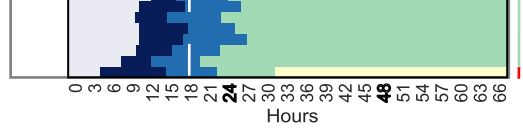

C

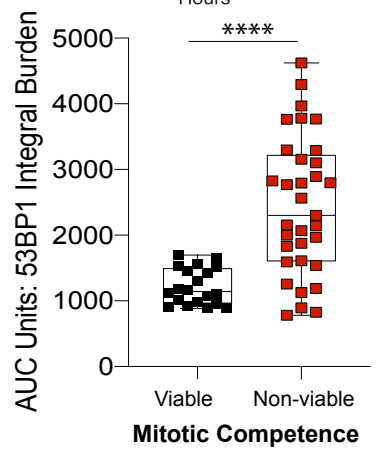

$20 \%$

$6 \%$

$43 \%$

$17 \%$
$6 \%$

$6 \%$

$70 \%$

$14 \%$

$10 \%$
$6 \%$

$91 \%$ b

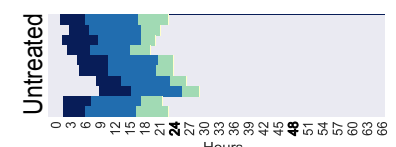

Figure 4
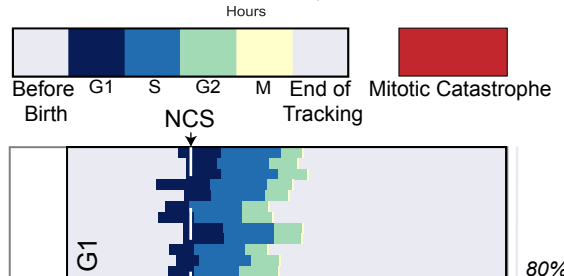

$40 \%$ วุ.

*3.

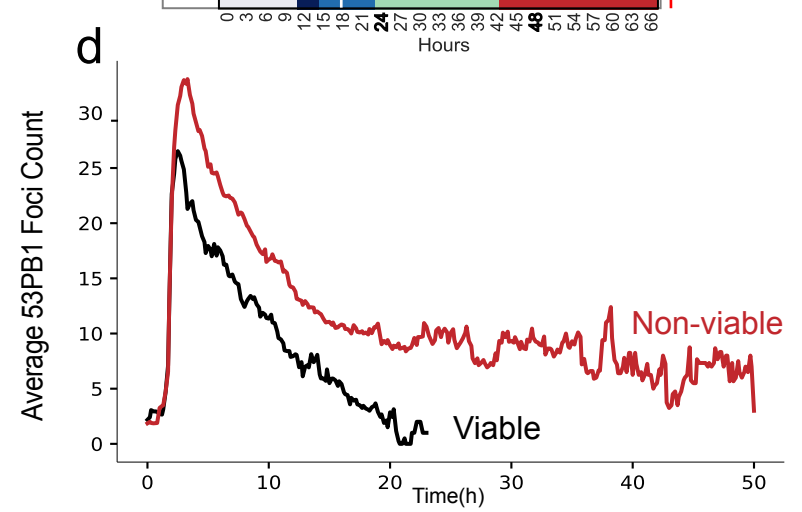


Figure 4 | Inhibition of DNA-PK induces catastrophic mitoses in p53-deficient cells. a, Cell cycle outcome analyses for si-control treated RPE1, dashed white line indicates drug addition, each row is an individual cell ( $n=60$ cells for NCS and $n=60$ cells for NCS+DNA-PKi treatment). Colored bars indicate different phases of the cell cycle, legend shown with no treatment control for comparison. Cells with red bars at the end of mitosis indicate terminal cell cycle event (mitotic catastrophe or apoptosis). Event frequency is reported as a percentage on the right. Cells exposed in G1 vs. S cells are treated as separate cohorts. Fisher's exact test was performed between -/+ DNA-PKi cohorts using 2 outcome groups (viable, vs. non-viable (arrested cells + terminal outcomes). ${ }^{* * * *} p<0.0001$, n.s. $=$ non-significant $\mathbf{b}$, Cell cycle outcome analyses for si-TP53 treated RPE1, dashed line indicates drug addition, each row is an individual cell ( $n=60$ cells for NCS and $n=60$ cells for NCS+DNA-PKi treatment). c, AUC analysis of 53BP1 damage burden in viable vs. non-viable p53-deficient cells that were treated with NCS and DNAPKi. Statistical significance was calculated using a Mann-Whitney test comparing ranks. ${ }^{* * * *} p<0.0001 \mathrm{~d}$, Dynamics of 53BP1 foci burden p53-deficient RPE1 segregated by mitotic viability. The red line corresponds to mean 53BP1 foci burden for all p53-deficient cells treated with NCS and DNA-PKi that undergo catastrophic mitoses, black line indicates mean foci value for p53-deficient cells with NCS and DNA-PKi treatment that are viable post mitosis, ( $n=20$ viable cells and $n=33$ non-viable cells). 


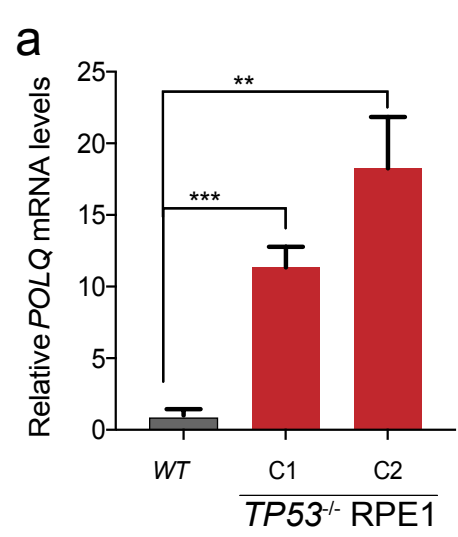

b

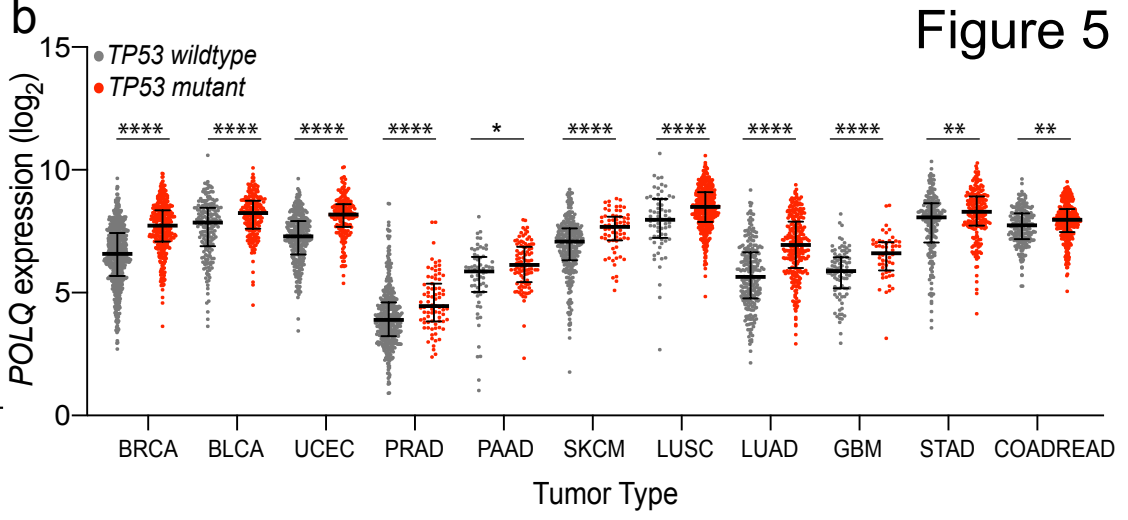

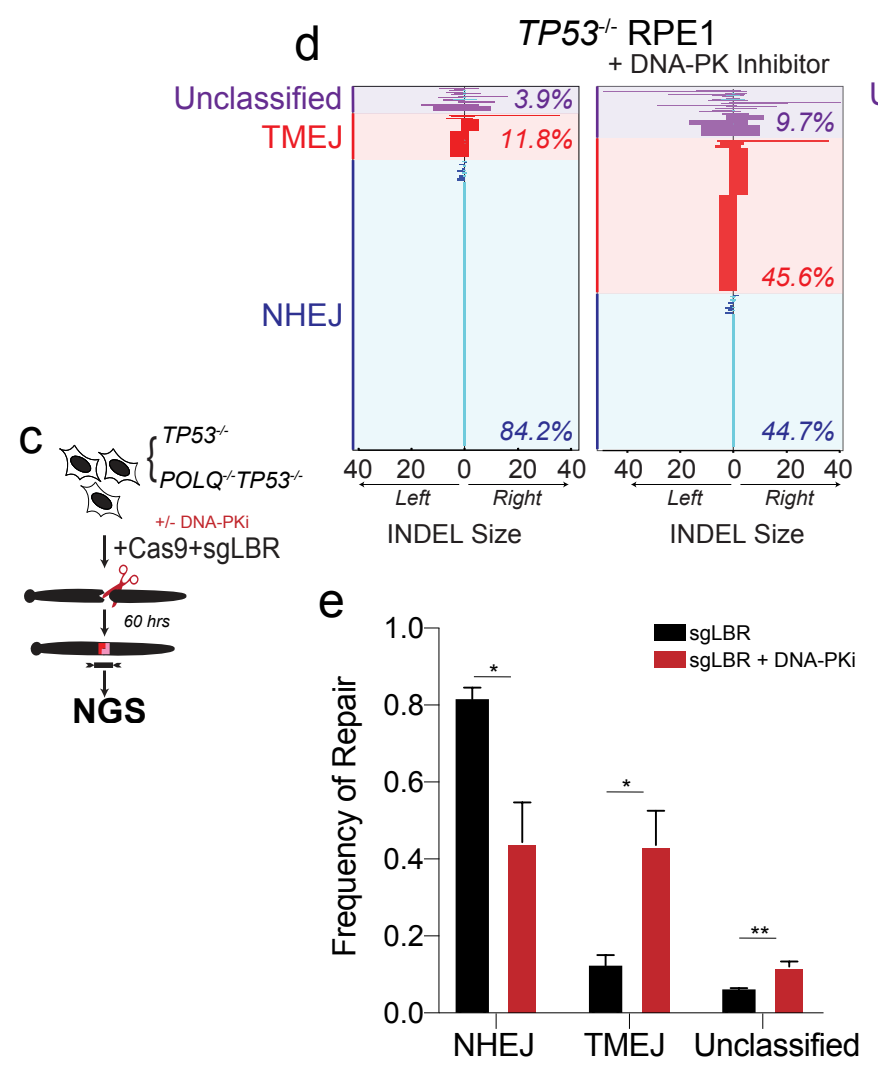
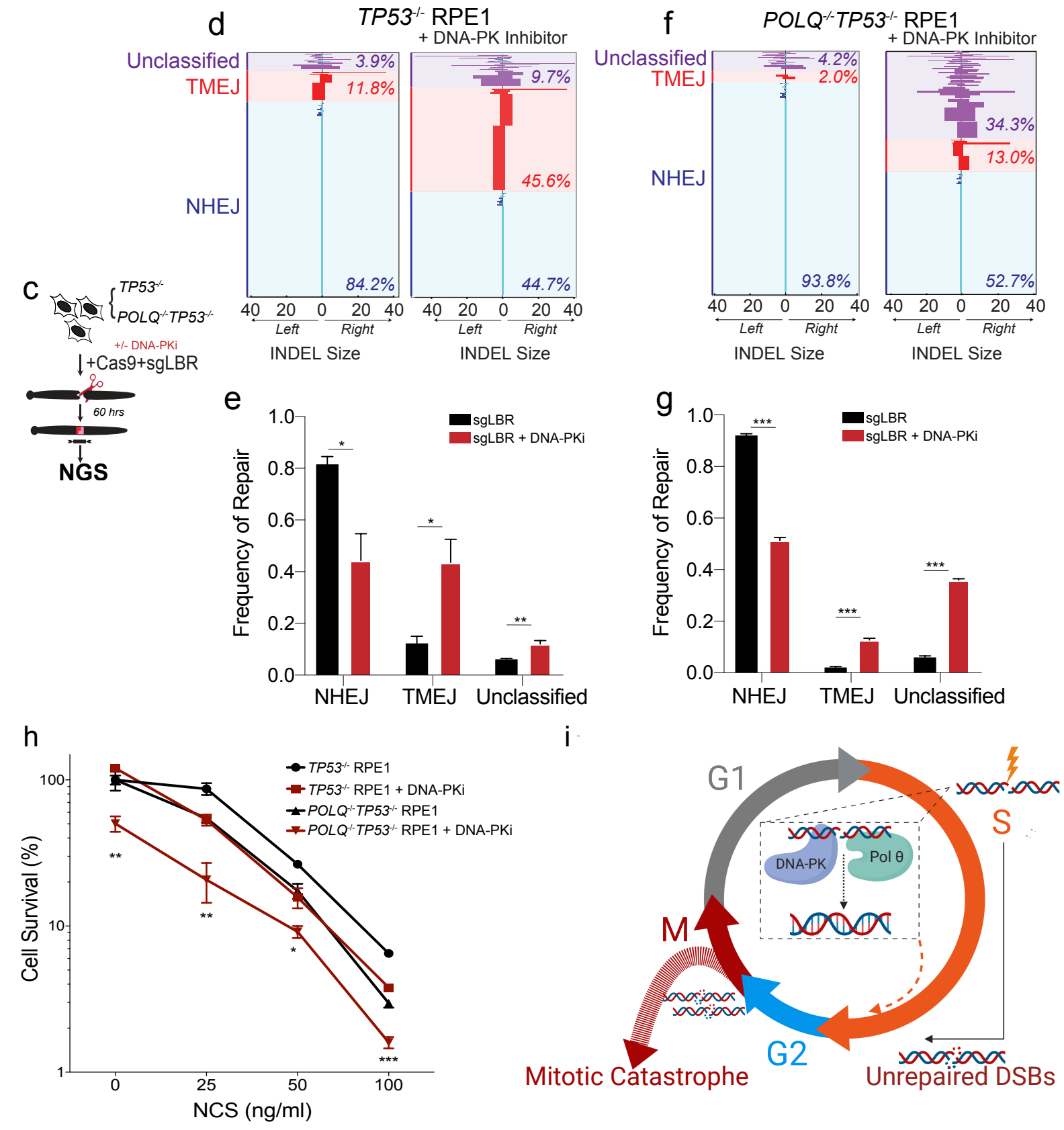
Figure 5 | p53-deficient cells utilize alternative end-joining pathways in the absence of active DNA-PK

a, RT-qPCR for POLQ mRNA levels in 2 TP53--RPE1 clones compared to WT RPE1. Significance was determined using two-tailed t-test. ${ }^{* * * *} p<0.0001,{ }^{* *} p<0.01$. b, $P O L Q$ gene expression depicted as log2 values of TP53 wild-type vs. mutant cancers across a subset of TCGA tumor types. Tumor labels follow TCGA labeling format. BRCA: breast cancer, BLCA: B-cell lymphoma, UCEC: uterine cancer, PRAD: Prostate cancer, PAAD: pancreatic cancer, SKCM: melanoma, LUSC: lung squamous cell cancer, LUAD: lung adenocarcinoma, GBM: glioblastoma multiforme, STAD: stomach cancer, and COADREAD: colorectal cancer. ${ }^{* * * *} p<0.0001,{ }^{* * *} p<0.001,{ }^{* *} p<0.01,{ }^{*} p<0.05$, as calculated by one-way ANOVA. c, Schematic depicting chromosomal break repair assay.

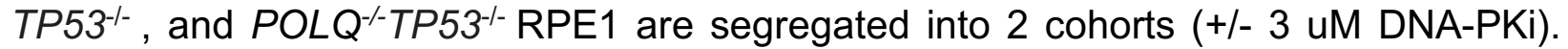
Cells are electroporated using Cas9-RNP-sgRNA-LBR and evaluated by next generation sequencing for break repair products at target locus. d, Horizontal bar chart representation of individual break repair products at $L B R$ locus in TP53-- RPE1 by NGS. Position 0 denotes $L B R$ locus cut site, with left and right positions denoting final INDEL size and orientation. Results are reported as average with SEM of $n=3$ independent biological replicates. e, Histogram of overall frequency of repair of NHEJ, TMEJ, and Unclassified products in TP53-- RPE1 with or without DNA-PKi treatment. f, Horizontal bar chart representation of individual break repair products at $\angle B R$ locus in $P O L Q^{-1-T P 53-}$ 1- RPE1 by NGS. Position 0 denotes $L B R$ locus cut site, with left and right positions denoting final INDEL size and orientation. Results are reported as average with SEM of $\mathrm{n}=3$ independent biological replicates. g, Histogram of overall frequency of repair of NHEJ, TMEJ, and Unclassified products in TP53-- RPE1 with or without DNA-PKi

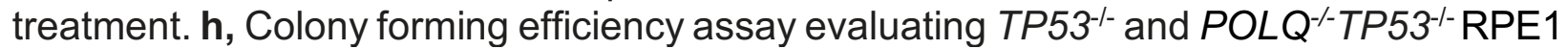
after treatment with NCS (at $25 \mathrm{ng} / \mathrm{ml}, 50 \mathrm{ng} / \mathrm{ml}$, and $100 \mathrm{ng} / \mathrm{ml}$ ) with or without $.5 \mathrm{uM}$ DNA-PKi, data shown are mean $+/$ - SEM $(n=3)$. Statistical significance assessed with student's two-tail test. ${ }^{* * *} p<0.001,{ }^{* *} p<0.01,{ }^{*} p<0.05$. in comparison to the survival

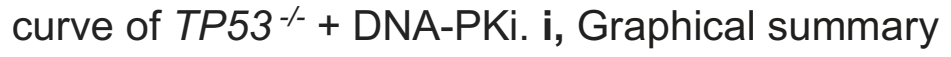




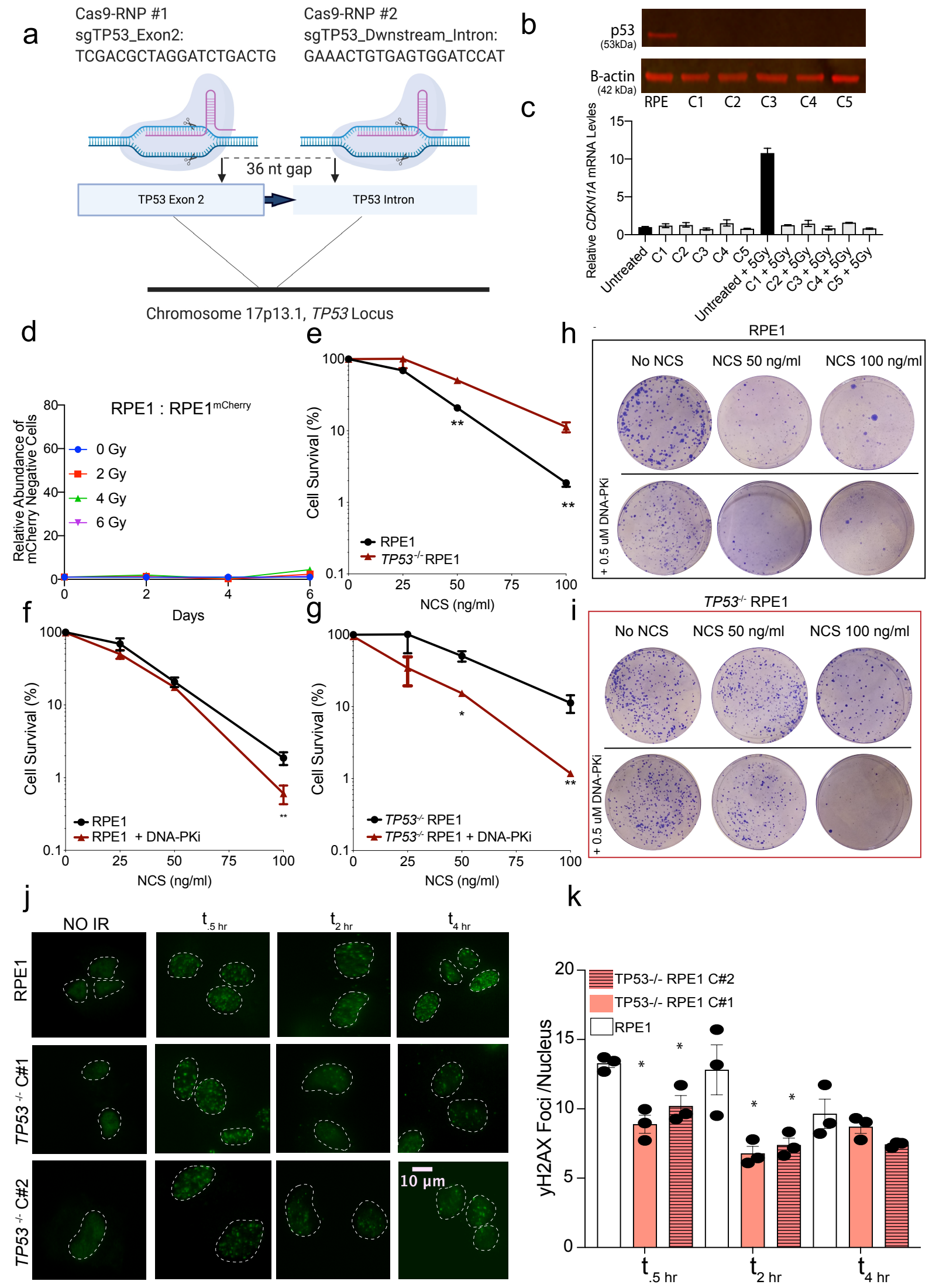

Supplementary Figure 1 
Supplementary Figure 1 | a, Schematic of CRISPR target locus in human TP53 gene. Two sgRNAs were designed to target sites in the terminal region of exon 2 (which encodes the p53 transactivating domain) and a site in the downstream intron with a 36 nucleotide (nt) gap. sgRNAs were complexed with Cas9 in the RNP system and electroporated into RPE1 cells. b, Western Blot of 5 selected single-cell clones that were profiled for p53 protein. c, Functional assay evaluating p53-dependent CDKN1A transcriptional responses to treatment of 5Gy IR. RNA from cells exposed to IR were harvested 6 hrs post treatment. d, Relative abundance of unlabeled RPE1 unlabelled over RPE1 ${ }^{\text {Cherry }}$ measured by Intellicyte high-throughput cytometry +/- SEM $(n=6)$ is shown, normalized to the untreated (0Gy) cohort at each time point. e, Clonogenic survival assays performed in RPE1 vs TP53-- RPE1 cells exposed to NCS. f, Clonogenic survival assays of RPE1 treated NCS +/- 0.5 uM DNA-PKi. Reported values are mean of $n=3$ replicates, and survival fraction was calculated by first calculating plating efficiency and normalizing it to the untreated samples. g, TP53 ${ }^{--}$RPE1 cells were treated with NCS +/0.5 uM DNA-PKi. $\mathbf{h}, \mathbf{i}$, Representative colony forming plates for $e$ and $f$ at NCS doses of 0,50 and $100 \mathrm{ng} / \mathrm{mL}+/-0.5 \mathrm{uM}$ DNA-PKi. Cell numbers for each conditions plated are the following: UT (500), NCS $50 \mathrm{ng} / \mathrm{ml}$ (2000), NCS $100 \mathrm{ng} / \mathrm{ml}$ (6000). j, Representative immunofluorescence images of $\mathrm{yH} 2 \mathrm{AX}$ foci in cells with indicated genotypes untreated (no IR) or treated with IR (5Gy) and collected at .5, 2, and $4 \mathrm{~h}$ after irradiation. $\mathbf{k}$, Quantification of $y H 2 A X$ foci. Data shown are mean ( $n=50$ cells per treatment condition) + - SEM $(n=3)$, and are consistent across two independent biological replicates. ${ }^{*} p<0.05$ by two-tailed Student's t-test. 
a

si-Control treated RPE1

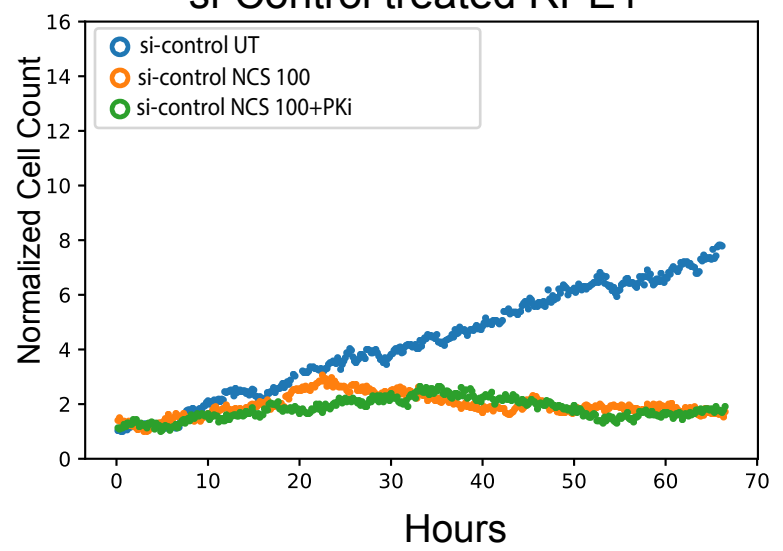

b

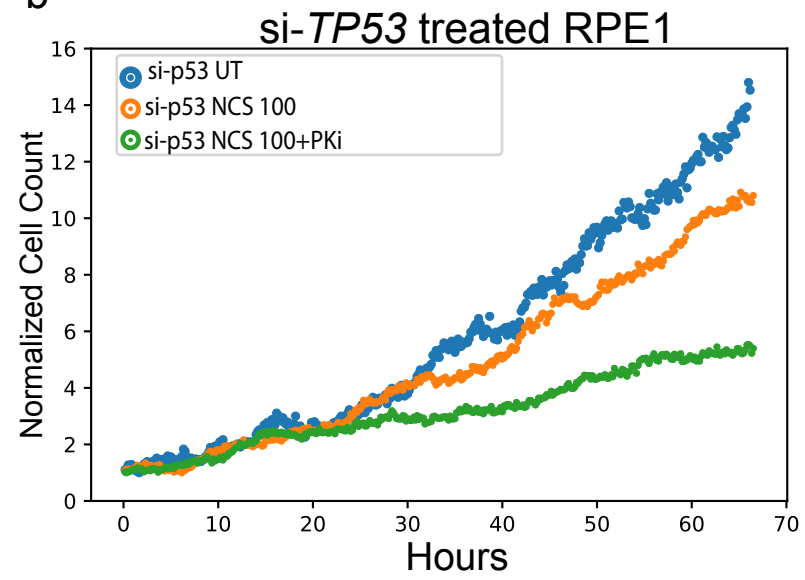

C

d

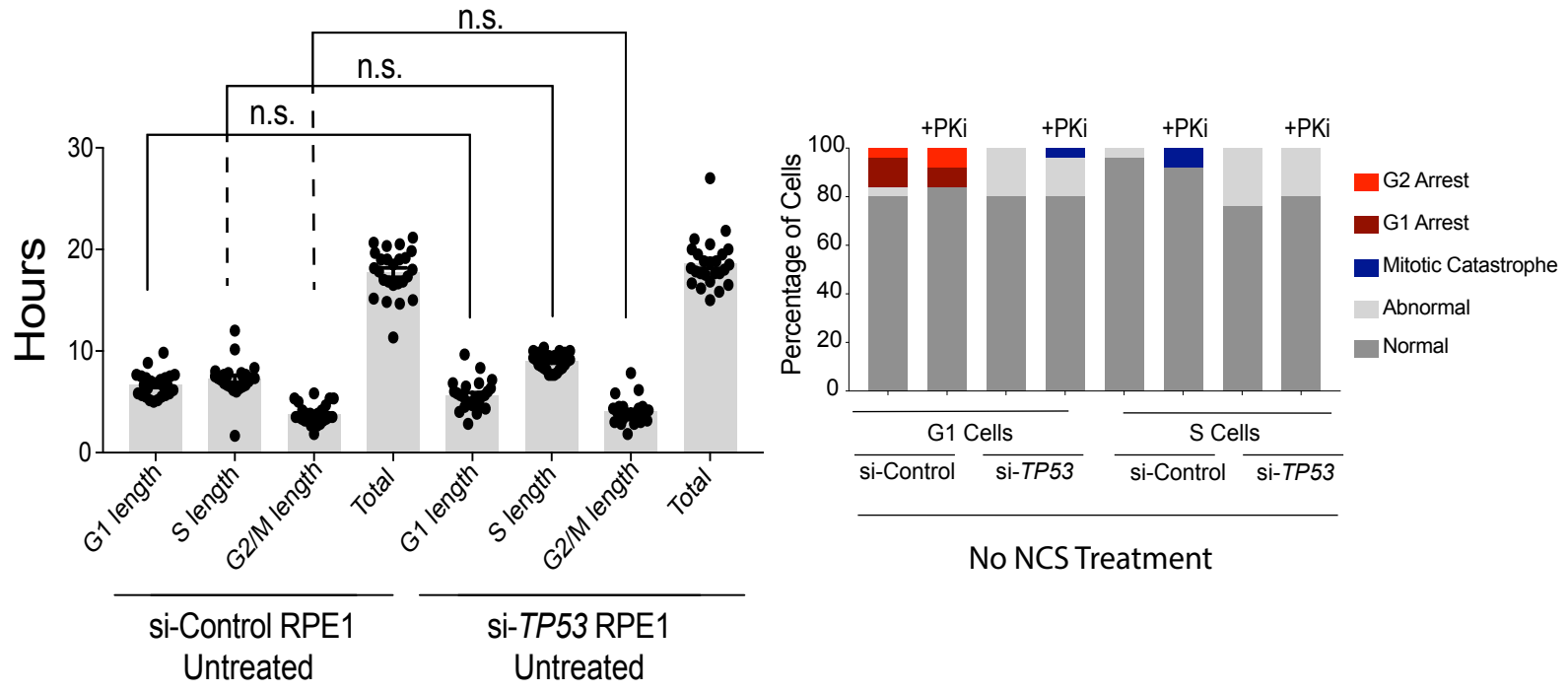

Supplementary Figure 2 
Supplementary Figure 2 | a, Quantification of cell proliferation from live-cell imaging experiments for si-Control treated RPE1. Cell counts were normalized to cell numbers at start of imaging. Here we show one representative imaging beacon for each treatment condition (untreated, NCS $100 \mathrm{ng} / \mathrm{ml}$ at 18 hours, and NCS $100 \mathrm{ng} / \mathrm{ml}+0.5 \mathrm{uM} \mathrm{DNA-PKi}$ at 18 hours). b, Cell proliferation counts for si-TP53 treated RPE1 over live-cell imaging. c, Analysis of RPE1 with no exposure to NCS (untreated) but received si-Control or siTP53 3 days prior to imaging. No significant differences are seen due to si-treatment alone. d, Quantification of baseline mitotic outcomes with no NCS exposure over the course of imaging. DNA-PKi treatment alone with no NCS showed no little to no additional effect on cells (Chi-squared analysis $\mathrm{P}>0.05$ for each condition). 
Please refer to the following file names for the Supplementary Figure 3 Video Files: Each video is a split screen of the same cell depicted in 2 channels: PCNA (left) and 53BP1 (right)

3a: NormalMitosis.mp4

3b: TransientG2Delay.mp4

3c: G1Arrest.mp4

Supplementary Figure 3 | a, Normal Mitosis: RPE1 Cell cycle representative of normal mitosis, with NCS treatment only. For all cells in this figure both the PCNA and the 53BP1 channels are shown as two individual movies. b, Transient G2 Delay: RPE1 cell cycle representative of a transient cell cycle delay in G2 (length of G2 is significantly prolonged in comparison to untreated cells). This cell was treated with NCS and DNA-PKi. c, G1 Arrest: RPE1 cell cycle representative of a permanent G1 arrest. This is a p53 proficient cell treated with NCS and DNA-PKi. 
a

Apoptosis
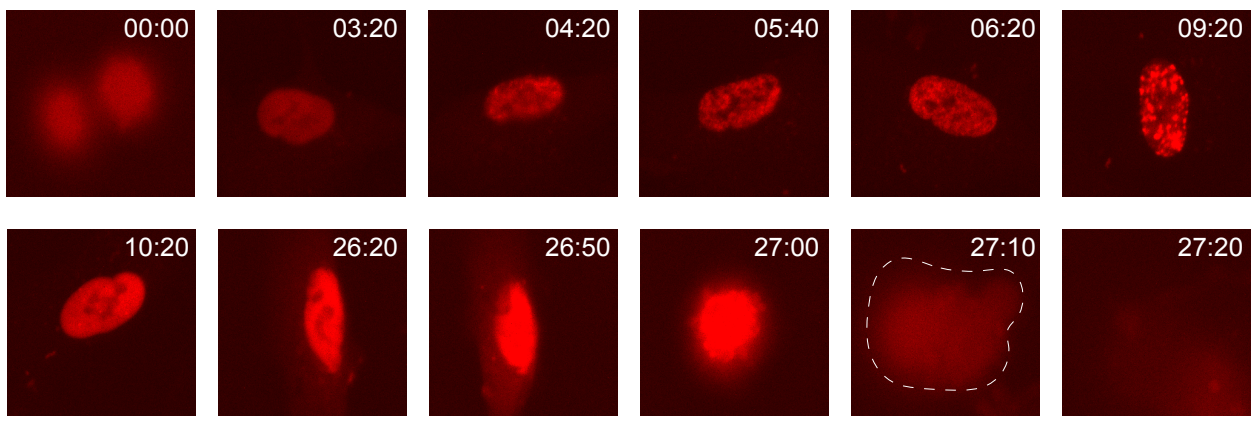

b

Mitotic Catastrophe
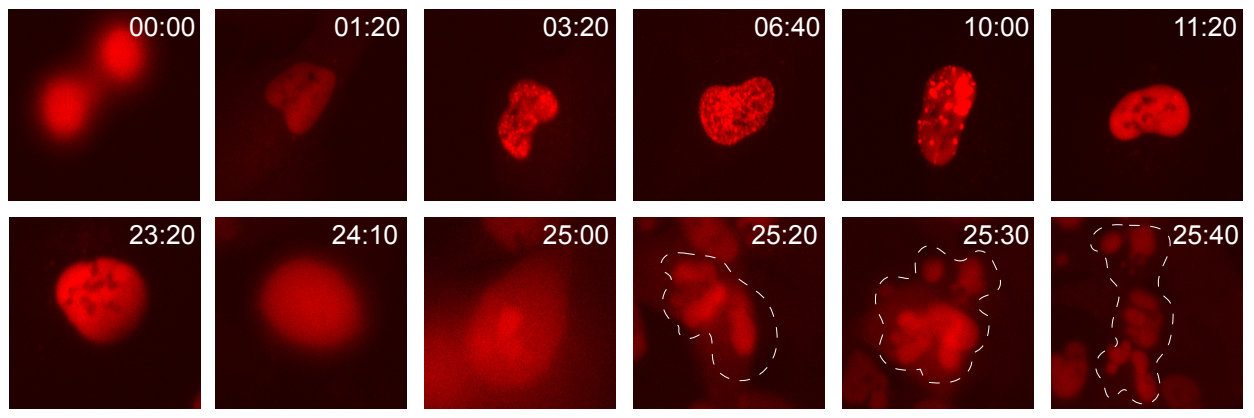

C

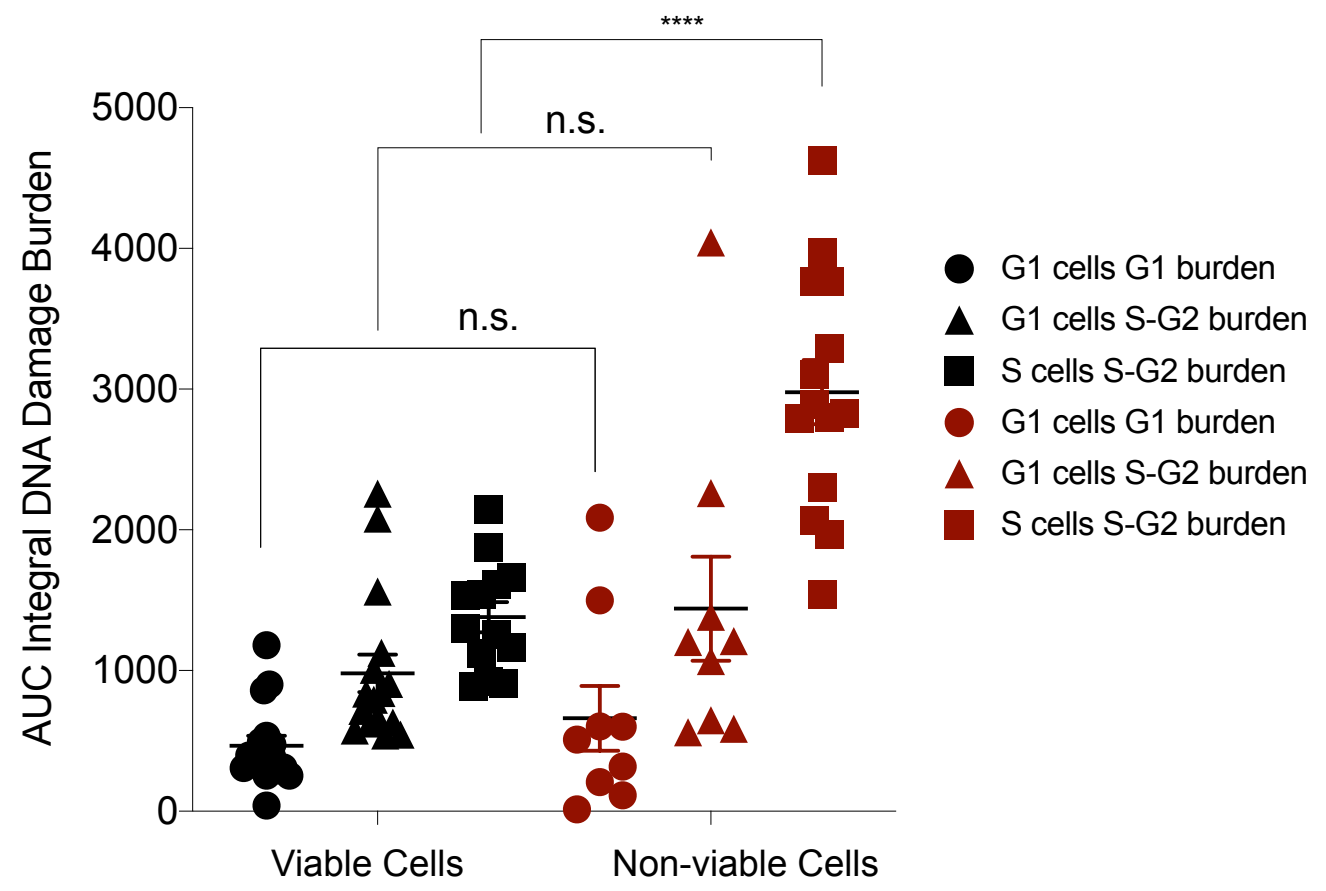

p53-Deficient RPE1 treated with NCS and DNA-PKi

Supplementary Figure 4 
Supplementary Figure $4 \mid$ a, Time stamped image sequence of apoptotic cell (PCNA channel shown). Cells that experienced nuclear degradation during cell cycle prior to mitosis were categorized as "apoptotic cells." In this sequence a cell in G2 experiences cell death at 27 hours post birth, with indication of mitotic attempt, with nuclear envelope collapse or presence of any daughter cells. $\mathbf{b}$, Time stamped image sequence of cell that experienced mitotic catastrophe (PCNA channel shown). Cell undergoes nuclear envelope collapse (24:10), and attempts mitosis, in subsequent images fragmentation of nucleus is clearly visible with no viable daughter cells present. Cell non-viability during mitosis was defined as mitotic catastrophe. c, Integral DNA damage burden for p53deficient cells treated with NCS $(100 \mathrm{ng} / \mathrm{ml})$ and DNA-PKi $(.5 \mathrm{uM})$ are calculated and segregated by viable (black) vs. non-viable outcomes (red). Legend indicates which phase of cell cycle the cells are in during drug exposure, followed by the phase for which the burden is calculated. Ex: $\mathrm{G} 1$ cells $\mathrm{G} 1$ burden = cells in $\mathrm{G} 1$ during drug exposure and total damage burden in $\mathrm{G} 1$. Area under the curve (AUC) analysis was performed by plotting 53BP1 foci counts over time for each cell and integrating burden over time. Statistical significance was determined using two-tailed Student's t-test. 
a

Cas9-RNP \#1 sgPOLO_PolDomain1: ACTACTCTCAGCTTGA

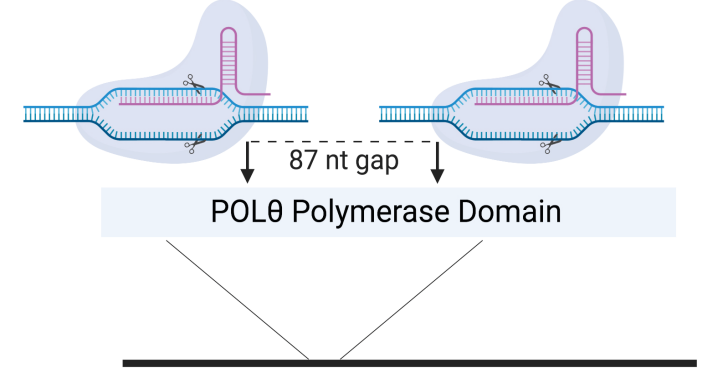

Chromosome 3q13.33, POL $\theta$ Locus

d

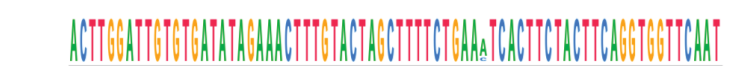

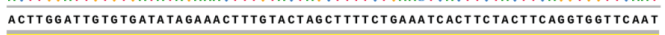

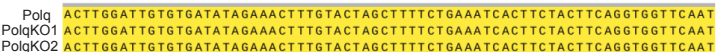

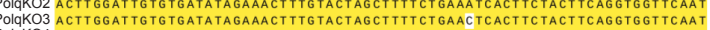

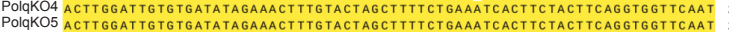

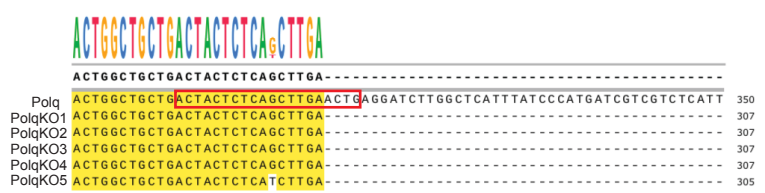

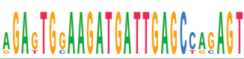
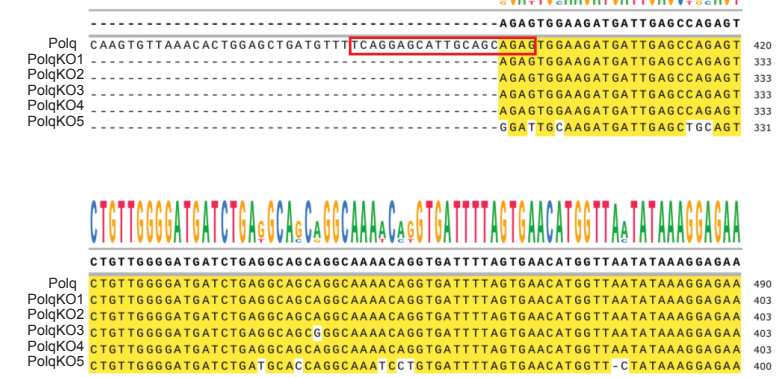

g

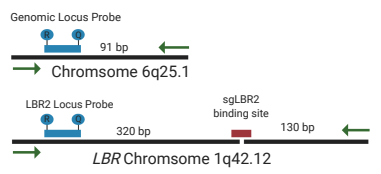

Digital PCR Detection Rate $=\#$ \#BR Locus Copies \# Genomic Locus Copies

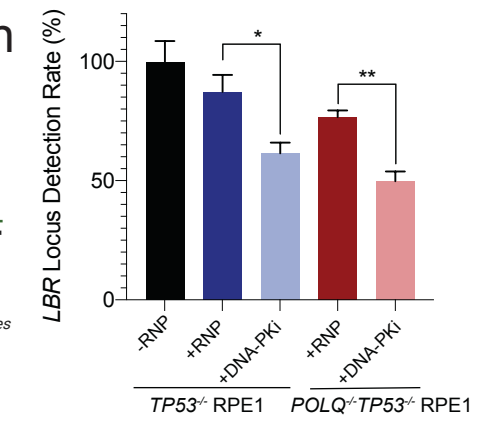

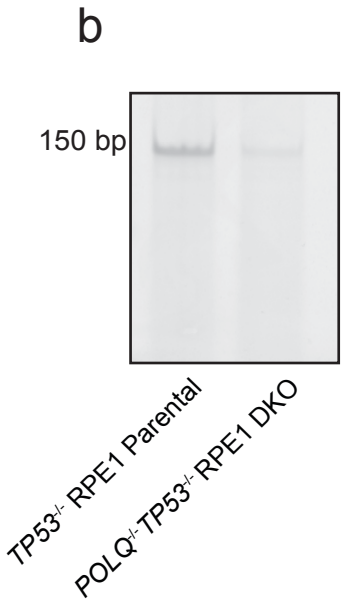

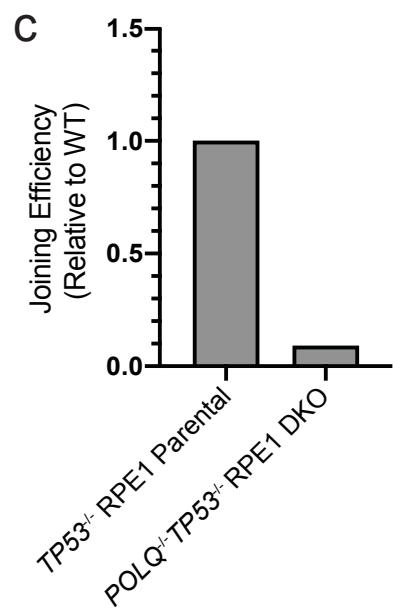

e

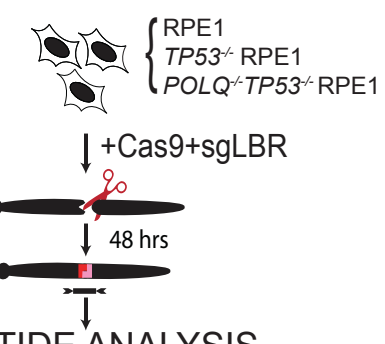

TIDE ANALYSIS
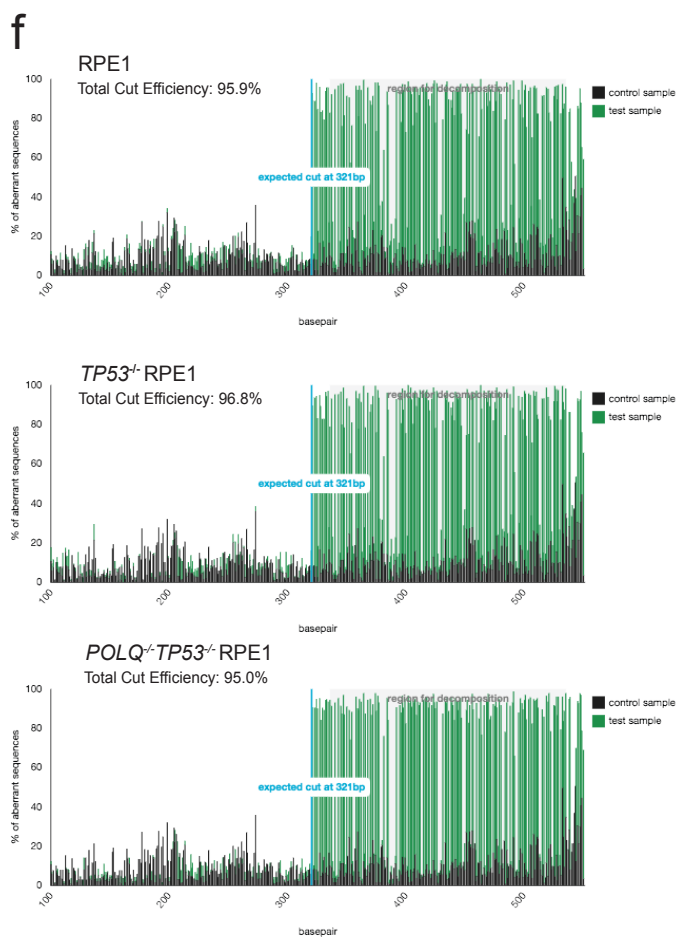

\section{Supplementary Figure 5}


Supplementary Figure 5 | a, Schematic of CRISPR target locus in human POLQ gene. Two sgRNAs were designed to target sites in the polymerase domain, with an 87 nucleotide (nt) gap. sgRNAs were complexed with Cas9 in RNP system and electroporated into RPE1 cells with a TP53\%- background to create double knockout cell line. b,c POLQ specific substrates were introduced into the TP53\% vs. POLQ ${ }^{-1}$ TP53\% DKO cells to assess repair efficiency. Products were amplified and characterized by electrophoresis and end joining efficiency was normalized to RPE1 with POLQ expression. d, Sanger sequencing analysis of CRISPR edited locus in $P O L Q^{-/}$TP5 $53^{-/}$ RPE1 clones. The locus of interest was PCR-amplified and cloned into a TOPO vector for sequencing analyses. Each line of sequence shown was derived from a different TOPO clone and aligned to show differences. The $P O L Q^{-/} T P 53^{-/}$clone has $87 \mathrm{bp}$ deletion resulting in frameshift mutations. Red boxes indicated sgRNAs used for the CRIPSR. e, Schematic showing evaluation of NGS samples by TIDE analysis for efficiency of cleavage at $\angle B R$ target site across cell lines. f, RPE1, TP53-- and POLQ ${ }^{-/} T P 53^{--}$RPE1 cutting efficiency, all three cell lines have comparable levels of cutting efficiency with sgLBR. g, Schematic depicting digital PCR method for assessing LBR detection rate. $\mathbf{h}$,

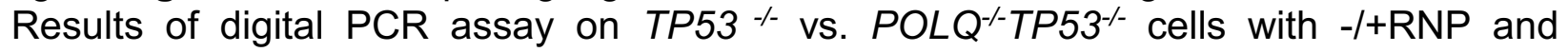
subsequently -/+ DNA-PKi (3uM). Average of 3 independent biological replicates are shown with SEM. Statistical significance was calculated using Multiple-t tests. ${ }^{*} p<0.05$, ${ }^{* *} \mathrm{p}<0.01$. 